\title{
The method for measuring piezooptic coefficients of crystals using a crystalline disk loaded along its diameter
}

\author{
Savaryn V., Skab I., Krupych O. and Vlokh R. \\ Institute of Physical Optics, 23 Dragomanov St., 79005 Lviv, Ukraine
}

Received: 23.04 .2012

\begin{abstract}
We develop a method for measuring piezooptic coefficients $\pi_{k k}$ $(k=4,5,6)$ for the crystals that belong to almost all of the point symmetry groups. The method is based on the studies of optical birefringence and optical indicatrix rotation angle under conditions of known spatial distribution of mechanical stress components in a crystalline disk compressed along its diameter. We present the relevant theoretical relations that describe piezooptic effect in crystals of all point symmetry groups.
\end{abstract}

Keywords: piezooptic effect, crystals, 2D stress state, crystalline disk

PACS: $78.20 . \mathrm{Hp}$

UDC: 535.55

\section{Introduction}

Piezooptic effect is a well-known phenomenon, of which the first experimental studies have been carried out by Brewster as early as in the beginning of the $19^{\text {th }}$ century $[1,2]$. The effect consists in changes of the refractive indices (or, equivalently, the optical impermeability coefficients $\Delta B_{i j}=\Delta\left(1 / n^{2}\right)_{i j}$ ) of an optical medium under the action of mechanical stresses $\sigma_{k l}$ :

$$
\Delta B_{i j}=B_{i j}-B_{i j}^{0}=\pi_{i j k l} \sigma_{k l} .
$$

Here $\pi_{i j k l}=\pi_{k m}$ is a fourth-rank piezooptic tensor, and $B_{i j}$ and $B_{i j}^{0}$ the optical impermeability tensors corresponding to stressed and free samples, respectively.

Usually the effect is studied after applying a uniaxial pressure to a sample. It is known that uniaxial loading of samples with parallelepiped shapes produces a barrel-shaped mechanical strain, due to friction forces that act between sample surfaces and substrates. This leads to appearance of all components of the stress tensor, of which spatial distribution is not known in advance [3]. As a consequence, the piezooptic coefficients are determined with high enough errors that exceed $\sim 30 \%$. In our recent work [4] we have developed a novel torsion method for studying the piezooptic coefficients. Under torsion, pure shear stress components appear in a crystalline sample, which depend linearly on the coordinates so that, in a convenient experimental geometry, one can measure the piezooptic coefficients $\pi_{k m}(k=1, \ldots, 3 ; m=4, \ldots, 6)$ with the errors reduced to $\sim 3 \%$. For determination of the coefficients $\pi_{k m}(k=1, \ldots, 3 ; m=1, \ldots, 3)$, we have developed the method based on the known 2D stress distribution in the sample subjected to a four-point bending stress [5]. It has been found that employing of such spatial distributions for determination of the piezooptic coefficients essentially increases the accuracy, when compared with the known techniques relying on application of uniaxial pressures [6,7]. It is worthwhile that the known 2D spatial distributions of the stress tensor components arise not only under bending or torsion of 
crystals. One of the methods for creating 2D stress states consists in compressing a crystalline disk along its diameter. The well-known mechanical model of a compressed crystalline disk has earlier been described, e.g., in the works [8, 9]. Moreover, our recent work [10] has clearly demonstrated some advantages of the technique based on disks compressed along their diameters, when being applied to high-accuracy determination of the piezooptic coefficients in $\mathrm{LiNbO}_{3}$ crystals. Notice that the method of the compressed disk can also be suitable for highly accurate determination of the coefficients $\pi_{k k}(k=4, \ldots, 6)$.

In the present work we will consider theoretical relations for the refractive indices, the birefringence and the optical indicatrix rotation angle for the crystals of different point symmetry groups, which hold true for the crystalline disks loaded along their diameters. This will be a basic point of the high-accurate method developed by us for measuring the piezooptic coefficients.

\section{General theoretical analysis}

In general, the matrix of the piezooptic tensor may be written as

\begin{tabular}{l|llllll} 
& $\sigma_{11}$ & $\sigma_{22}$ & $\sigma_{33}$ & $\sigma_{32}$ & $\sigma_{31}$ & $\sigma_{21}$ \\
\hline$\Delta B_{11}$ & $\pi_{11}$ & $\pi_{12}$ & $\pi_{13}$ & $\pi_{14}$ & $\pi_{15}$ & $\pi_{16}$ \\
$\Delta B_{22}$ & $\pi_{21}$ & $\pi_{22}$ & $\pi_{23}$ & $\pi_{24}$ & $\pi_{25}$ & $\pi_{26}$ \\
$\Delta B_{33}$ & $\pi_{31}$ & $\pi_{32}$ & $\pi_{33}$ & $\pi_{34}$ & $\pi_{35}$ & $\pi_{36}$ \\
$\Delta B_{32}$ & $\pi_{41}$ & $\pi_{42}$ & $\pi_{43}$ & $\pi_{44}$ & $\pi_{45}$ & $\pi_{46}$ \\
$\Delta B_{31}$ & $\pi_{51}$ & $\pi_{52}$ & $\pi_{53}$ & $\pi_{54}$ & $\pi_{55}$ & $\pi_{56}$ \\
$\Delta B_{21}$ & $\pi_{61}$ & $\pi_{62}$ & $\pi_{63}$ & $\pi_{64}$ & $\pi_{65}$ & $\pi_{66}$
\end{tabular}

In order to describe our approach, let us consider as an example a disk-shaped sample prepared from the crystals which belong to the point symmetry group $3 \mathrm{~m}$ [10]. The faces of the disk are perpendicular to the $Z$ axis and the loading force $P_{2}$ is applied along the $Y$ axis. The following components of the stress tensor remain nonzero for this experimental geometry:

$$
\begin{aligned}
& \sigma_{1}=-\frac{2 P_{2}}{\pi d}\left[\frac{(R-Y) X^{2}}{\left(X^{2}+(R-Y)^{2}\right)^{2}}+\frac{(R+Y) X^{2}}{\left(X^{2}+(R+Y)^{2}\right)^{2}}-\frac{1}{2 R}\right], \\
& \sigma_{2}=-\frac{2 P_{2}}{\pi d}\left[\frac{(R-Y)^{3}}{\left(X^{2}+(R-Y)^{2}\right)^{2}}+\frac{(R+Y)^{3}}{\left(X^{2}+(R+Y)^{2}\right)^{2}}-\frac{1}{2 R}\right], \\
& \sigma_{6}=\frac{2 P_{2}}{\pi d}\left[\frac{(R-Y)^{2} X}{\left(X^{2}+(R-Y)^{2}\right)^{2}}+\frac{(R+Y)^{2} X}{\left(X^{2}+(R+Y)^{2}\right)^{2}}\right] .
\end{aligned}
$$

where $d$ denotes the thickness of the disk and $R$ its radius. Then the optical indicatrix equation becomes as follows:

$$
\left(B_{11}+\pi_{11} \sigma_{1}+\pi_{12} \sigma_{2}\right) X^{2}+\left(B_{11}+\pi_{12} \sigma_{1}+\pi_{11} \sigma_{2}\right) Y^{2}+2\left(\pi_{11}-\pi_{12}\right) \sigma_{6} X Y=1 .
$$

Application of mechanical stresses in this geometry would lead to changes in both the birefringence and the optical indicatrix rotation angle:

$$
\begin{aligned}
& \Delta n_{12}=-\frac{1}{2} n_{o}^{3}\left(\pi_{11}-\pi_{12}\right) \sqrt{\left(\sigma_{1}-\sigma_{2}\right)^{2}+4 \sigma_{6}^{2}}, \\
& \tan 2 \zeta_{3}=\frac{2\left(\pi_{11}-\pi_{12}\right) \sigma_{6}}{\left(B_{1}+\pi_{11} \sigma_{1}+\pi_{12} \sigma_{2}\right)-\left(B_{1}+\pi_{12} \sigma_{1}+\pi_{11} \sigma_{2}\right)}=\frac{2 \sigma_{6}}{\left(\sigma_{1}-\sigma_{2}\right)} .
\end{aligned}
$$


In particular, we have $\sigma_{6}=0$ along the diameter $X=0$ (i.e., along the diameter parallel to the loading force vector), while the components $\sigma_{1}$ and $\sigma_{2}$ are equal to

$$
\begin{aligned}
\sigma_{1} & =\frac{P_{2}}{\pi d R}, \\
\sigma_{2} & =-\frac{2 P_{2}}{\pi d}\left[\frac{2 R}{R^{2}-Y^{2}}-\frac{1}{2 R}\right] .
\end{aligned}
$$

The birefringence along the diameter is given by the relation

$$
\Delta n_{12}=-\frac{1}{2} n_{o}^{3}\left(\pi_{11}-\pi_{12}\right)\left(\sigma_{1}-\sigma_{2}\right)=-n_{o}^{3}\left(\pi_{11}-\pi_{12}\right) \frac{2 R P_{2}}{\pi d\left(R^{2}-Y^{2}\right)},
$$

while the angle of optical indicatrix rotation is equal to zero (see Eq. (8)). As a consequence, the coefficient $\pi_{66}=\pi_{11}-\pi_{12}$ can, in principle, be determined experimentally using a nonlinear dependence of the birefringence on the $Y$ coordinate.

\subsection{Cubic crystals}

The relations for the optical birefringence and the optical indicatrix rotation angle obtained by us for the disk-shaped crystalline samples made of cubic crystals are presented in Table 1. Since all of the three crystallographic axes of crystals belonging to the cubic system are identical from the viewpoint of the piezooptic effect, we will analyse the induced birefringence and the optical indicatrix rotation only for the $Z$-cut disk loaded along its diameter parallel to the $Y$ axis. The dependences of the refractive indices on the $Y$ coordinate (i.e., along the diameter of loading force) for the crystals of the symmetry groups $432, \overline{4} 3 \mathrm{~m}$ and $\mathrm{m} 3 \mathrm{~m}$ may be written as

$$
n_{1}=n_{o}-\frac{1}{2} n_{o}^{3} \frac{P_{2}}{\pi d}\left\{\frac{1}{R} \pi_{11}-\left[\frac{4 R}{R^{2}-Y^{2}}-\frac{1}{R}\right] \pi_{12}\right\}, \quad n_{2}=n_{o}-\frac{1}{2} n_{o}^{3} \frac{P_{2}}{\pi d}\left\{\frac{1}{R} \pi_{12}-\left[\frac{4 R}{R^{2}-Y^{2}}-\frac{1}{R}\right] \pi_{11}\right\}
$$

where $n_{o}$ is the initial (unperturbed) refractive index. The induced birefringence for this case reads as

$$
\Delta n_{12}=-n_{o}^{3} \frac{P_{2}}{\pi d} \frac{2 R}{R^{2}-Y^{2}}\left(\pi_{11}-\pi_{12}\right),
$$

while the angle of optical indicatrix rotation should be equal to zero along the $Y$ direction. Using Eq. (13) and the spatial distribution of the induced birefringence along the $Y$ direction obtained experimentally one can get the difference of the piezooptic coefficients $\pi_{11}-\pi_{12}$. The same difference can be determined under the condition $Y=0$. In this case the induced birefringence is given by

$$
\Delta n_{12}=-n_{o}^{3}\left(\pi_{11}-\pi_{12}\right) \frac{2 P_{2} R}{\pi d}\left[\frac{R^{2}-X^{2}}{\left(X^{2}+R^{2}\right)^{2}}\right],
$$

while the optical indicatrix rotation angle is equal to

$$
\tan 2 \zeta_{3}=\frac{2 \pi_{44}}{\left(\pi_{11}-\pi_{12}\right)} \frac{R X}{R^{2}-X^{2}} .
$$

Therefore the coefficient $\pi_{44}$ can be determined after measuring the dependence of the optical indicatrix rotation angle upon the $X$ coordinate and using the $\pi_{11}-\pi_{12}$ value known from the experiments described above (see Eqs. (13) and (14)). 


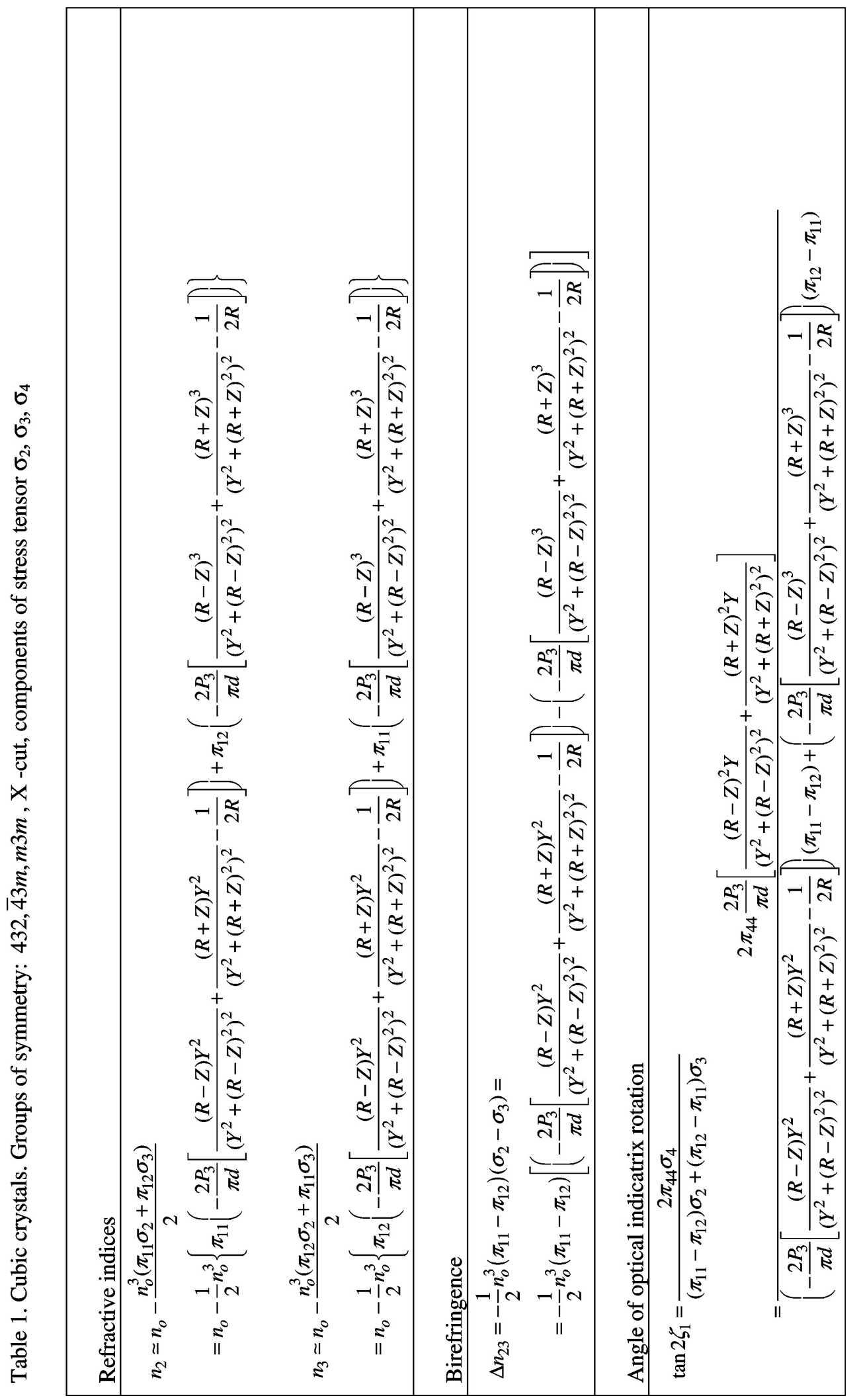




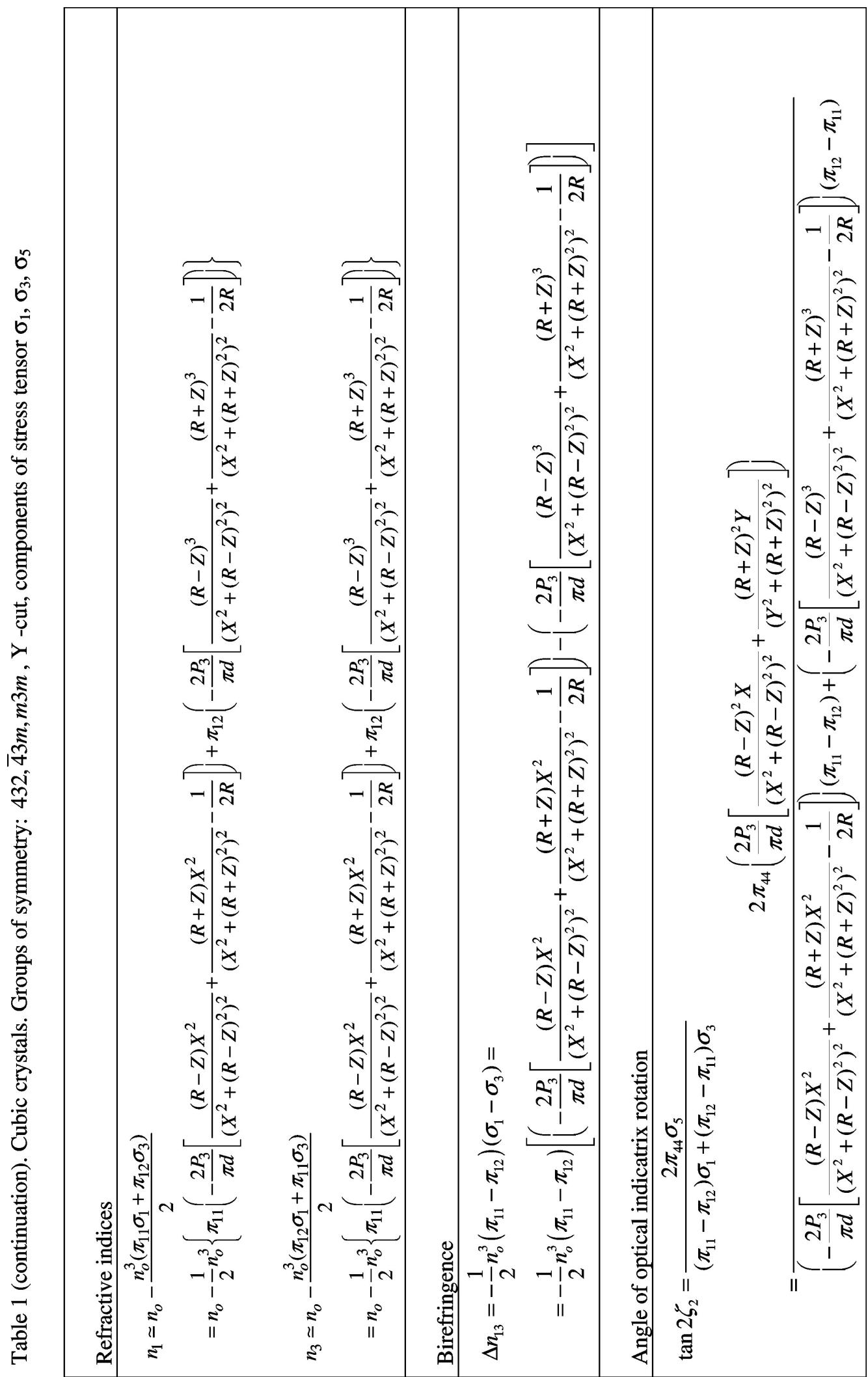




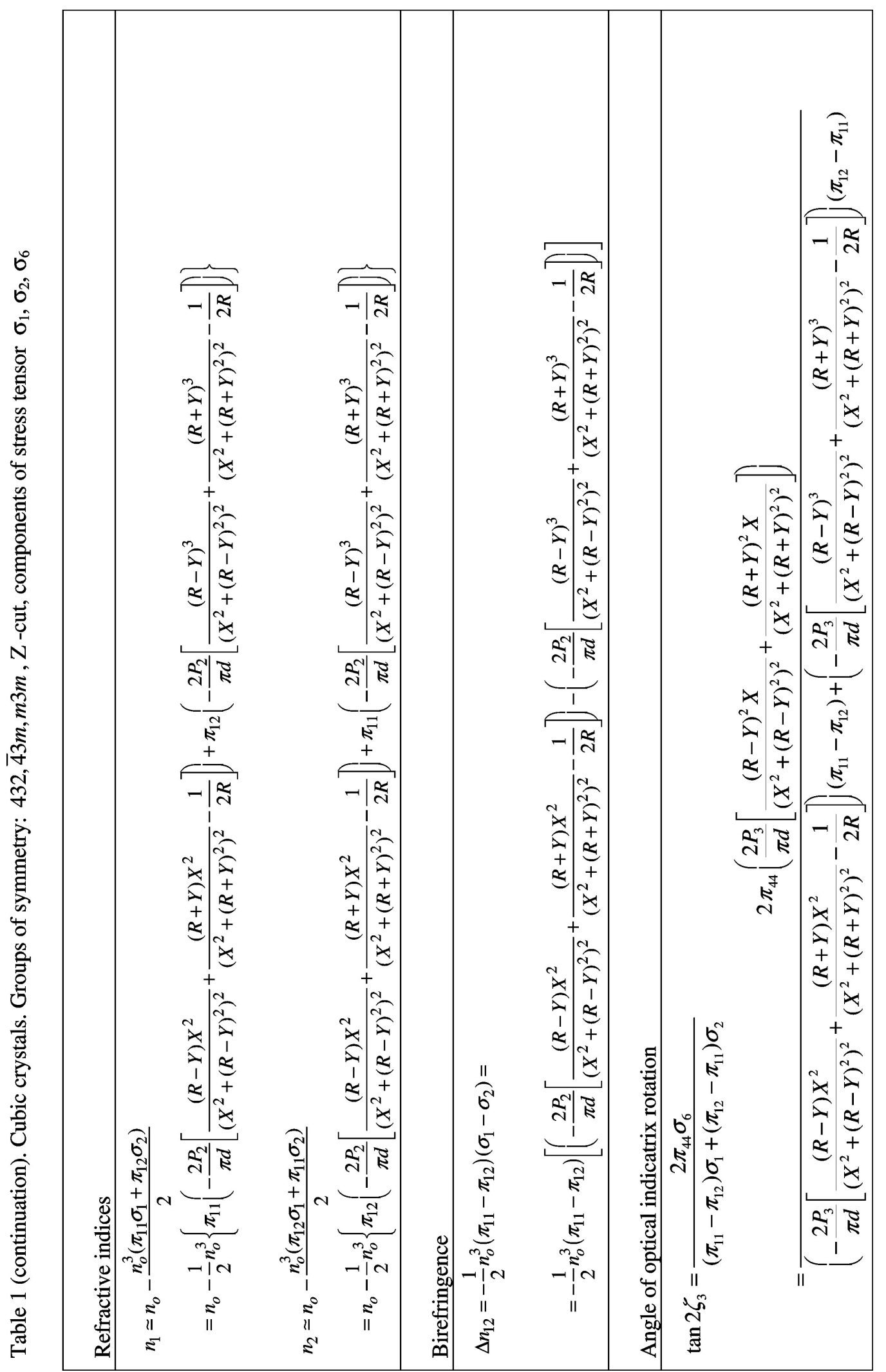

Ukr. J. Phys. Opt. 2012, V13, №2 


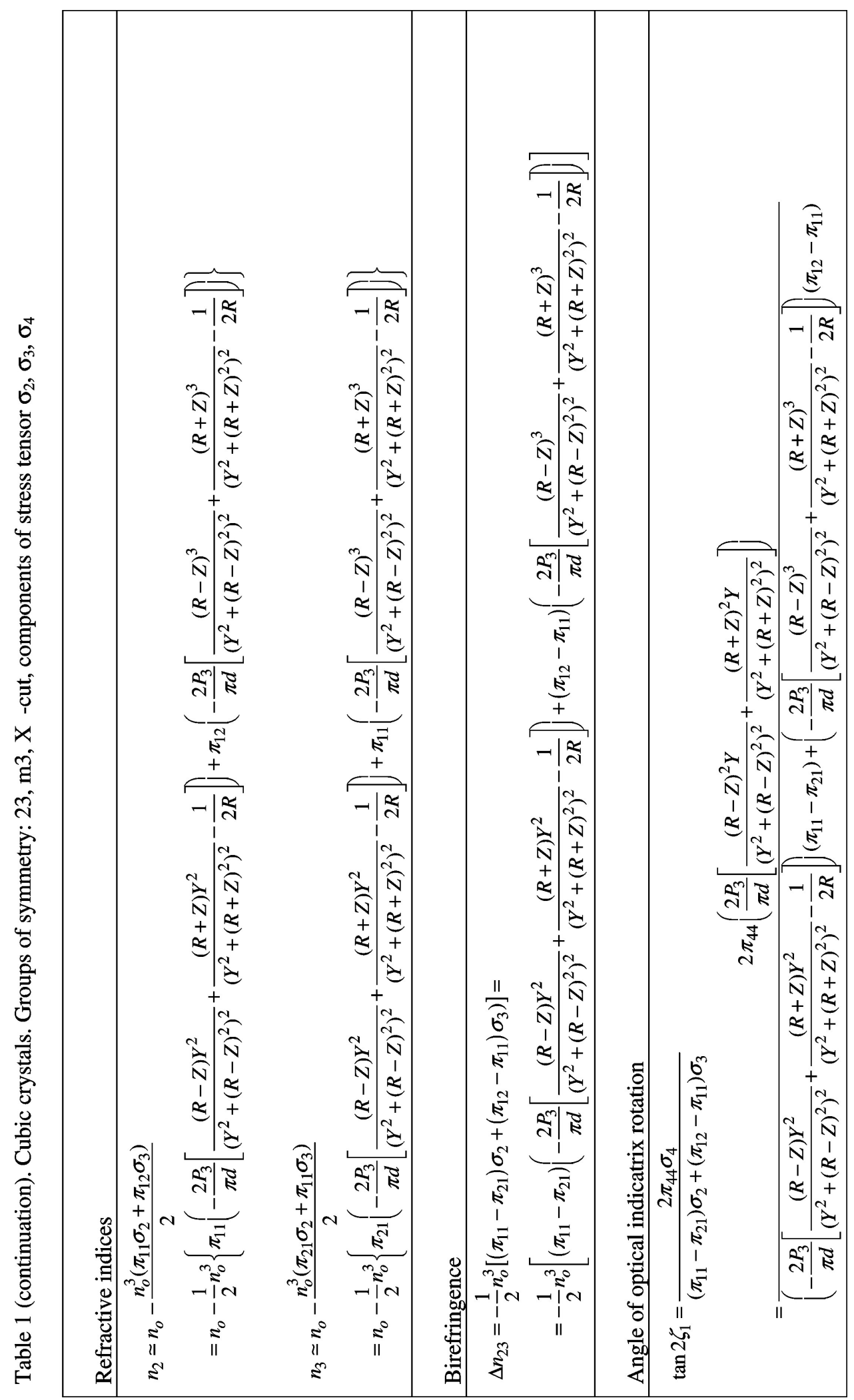




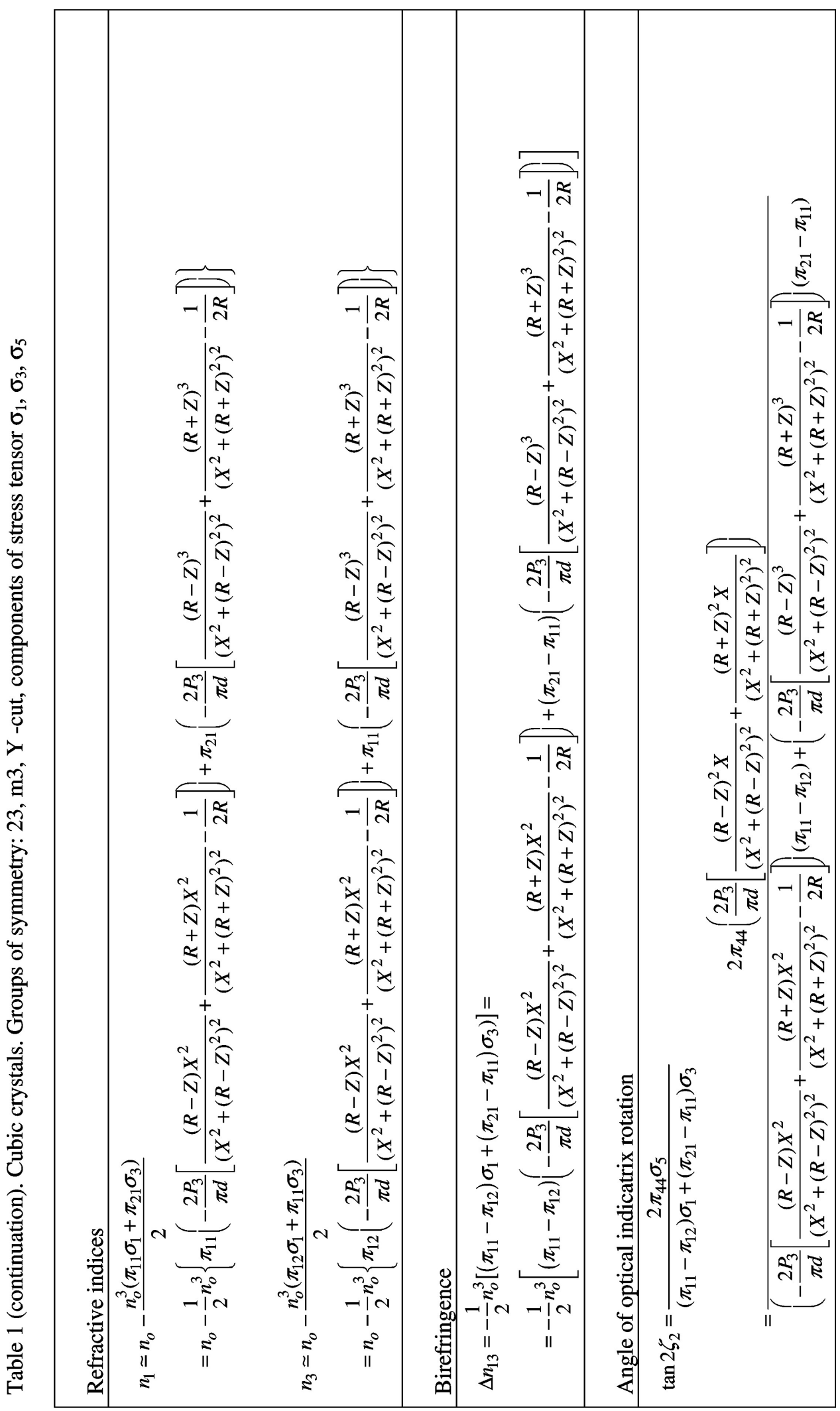

Ukr. J. Phys. Opt. 2012, V13, №2 


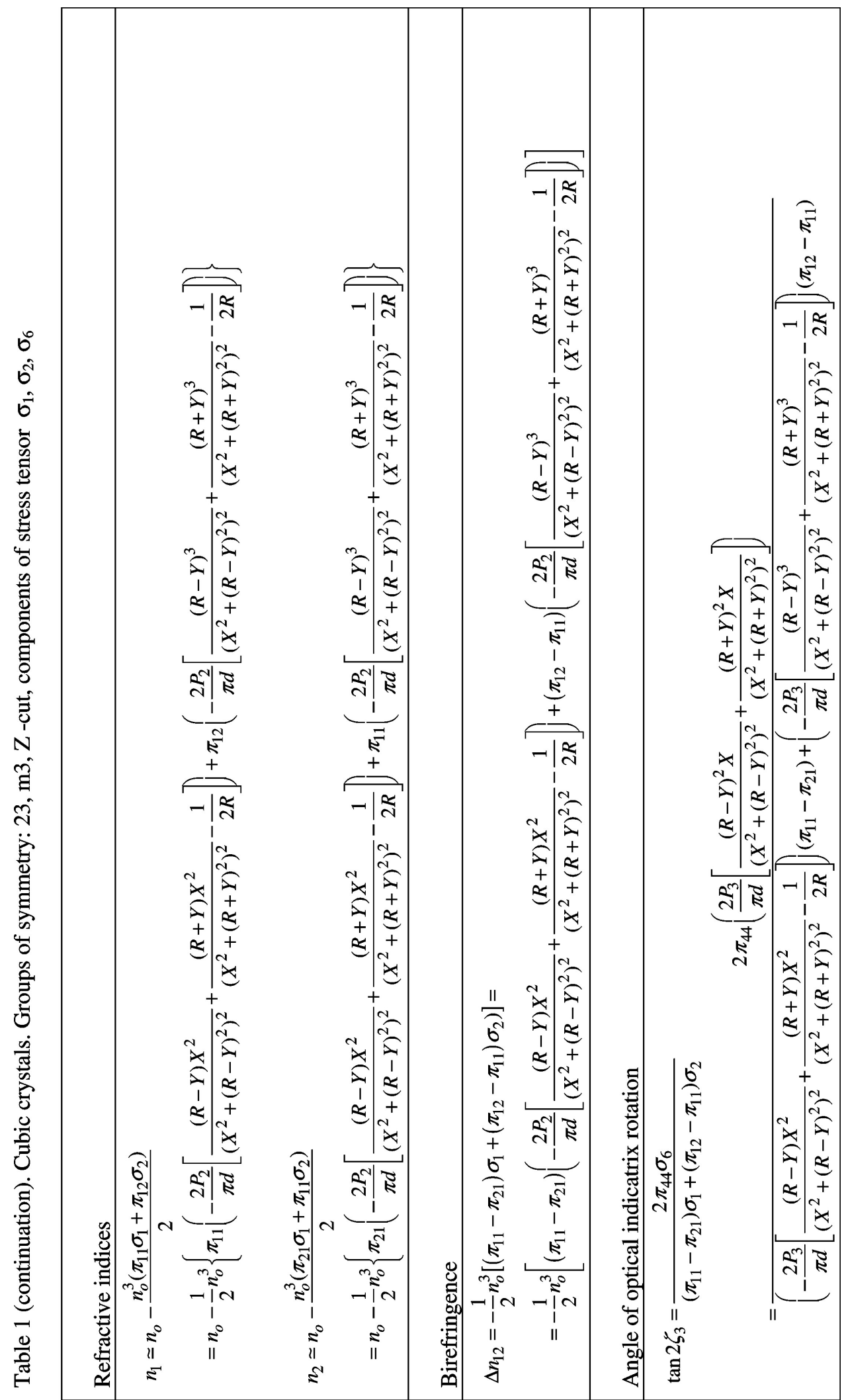


For the crystals belonging to the groups $\mathrm{m} 3$ and 23 , one can easily derive the induced birefringence under the same conditions (i.e., the loading force $P_{2}$ applied). For the case of $X=0$ we have

$$
\Delta n_{12}=-n_{o}^{3} \frac{P_{2}}{\pi d}\left[\frac{1}{2 R}\left(\pi_{12}-\pi_{21}\right)+\frac{2 R}{R^{2}-Y^{2}}\left(\pi_{11}-\pi_{12}\right)\right] .
$$

Given the condition of $\pi_{12}=\pi_{21}$, which is valid for the groups $432, \overline{4} 3 \mathrm{~m}$ and $\mathrm{m} 3 \mathrm{~m}$ just analysed, one can reduce Eq. (16) to Eq. (13). The birefringence in the centre of the disk is given by the relation

$$
\Delta n_{12}=-n_{o}^{3} \frac{P_{2}}{\pi d} \frac{1}{2 R}\left[4 \pi_{11}-3 \pi_{12}-\pi_{21}\right] .
$$

Eq. (17) may be employed for determining the combination of coefficients $4 \pi_{11}-3 \pi_{12}-\pi_{21}$. Notice that the angle of optical indicatrix rotation is equal to zero along the $Y$ axis.

The birefringence under the condition of $Y=0$ is as follows:

$$
\Delta n_{12}=-\frac{1}{2} n_{o}^{3} \frac{2 P_{2}}{\pi d}\left[\frac{2 R X^{2}}{\left(X^{2}+R^{2}\right)^{2}}\left(\pi_{21}-\pi_{11}\right)+\frac{2 R^{3}}{\left(X^{2}+R^{2}\right)^{2}}\left(\pi_{11}-\pi_{12}\right)+\frac{1}{2 R}\left(\pi_{12}-\pi_{21}\right)\right] .
$$

Using this formula, one can find a combination of piezooptic coefficients appearing in the round brackets of Eq. (18). Inserting this combination into the relation for the optical indicatrix rotation angle,

$$
\tan 2 \zeta_{3}=\frac{2 \pi_{44} \frac{2 R^{2} X}{\left(X^{2}+R^{2}\right)^{2}}}{\frac{2 R X^{2}}{\left(X^{2}+R^{2}\right)^{2}}\left(\pi_{21}-\pi_{11}\right)+\frac{2 R^{3}}{\left(X^{2}+R^{2}\right)^{2}}\left(\pi_{11}-\pi_{12}\right)+\frac{1}{2 R}\left(\pi_{12}-\pi_{21}\right)},
$$

one can evaluate the piezooptic coefficient $\pi_{44}$.

\subsection{Hexagonal crystals}

For the crystals that belong to the point symmetry groups $622,6 \mathrm{~mm}, \overline{6} \mathrm{~m} 2$ and $6 / \mathrm{mmm}$, the induced birefringence for the $Z$-cut crystalline disk loaded by the force $P_{2}$ along the $Y$ axis may be expressed as (see Table 2)

$$
\Delta n_{12}=-n_{o}^{3} \frac{P_{2}}{\pi d}\left(\pi_{11}-\pi_{12}\right) \frac{2 R}{R^{2}-Y^{2}}
$$

where $n_{o}$ denotes the ordinary refractive index. This enables determining the piezooptic difference $\pi_{11}-\pi_{12}$. The same parameter can be obtained under the condition $Y=0$. The birefringence along the $X$ axis reads as

$$
\Delta n_{12}=n_{o}^{3} \frac{2 R P_{2}}{\pi d}\left(\pi_{11}-\pi_{12}\right)\left\{\frac{X^{2}-R^{2}}{\left(X^{2}+R^{2}\right)^{2}}\right\} .
$$

The optical indicatrix rotation angle is equal to zero at $X=0$, while for the case of $Y=0$ it is given by

$$
\tan 2 \zeta_{3}=\frac{2 R X}{R^{2}-X^{2}}
$$

thus depending only on the geometrical parameters of the disk and the coordinate $X$. 


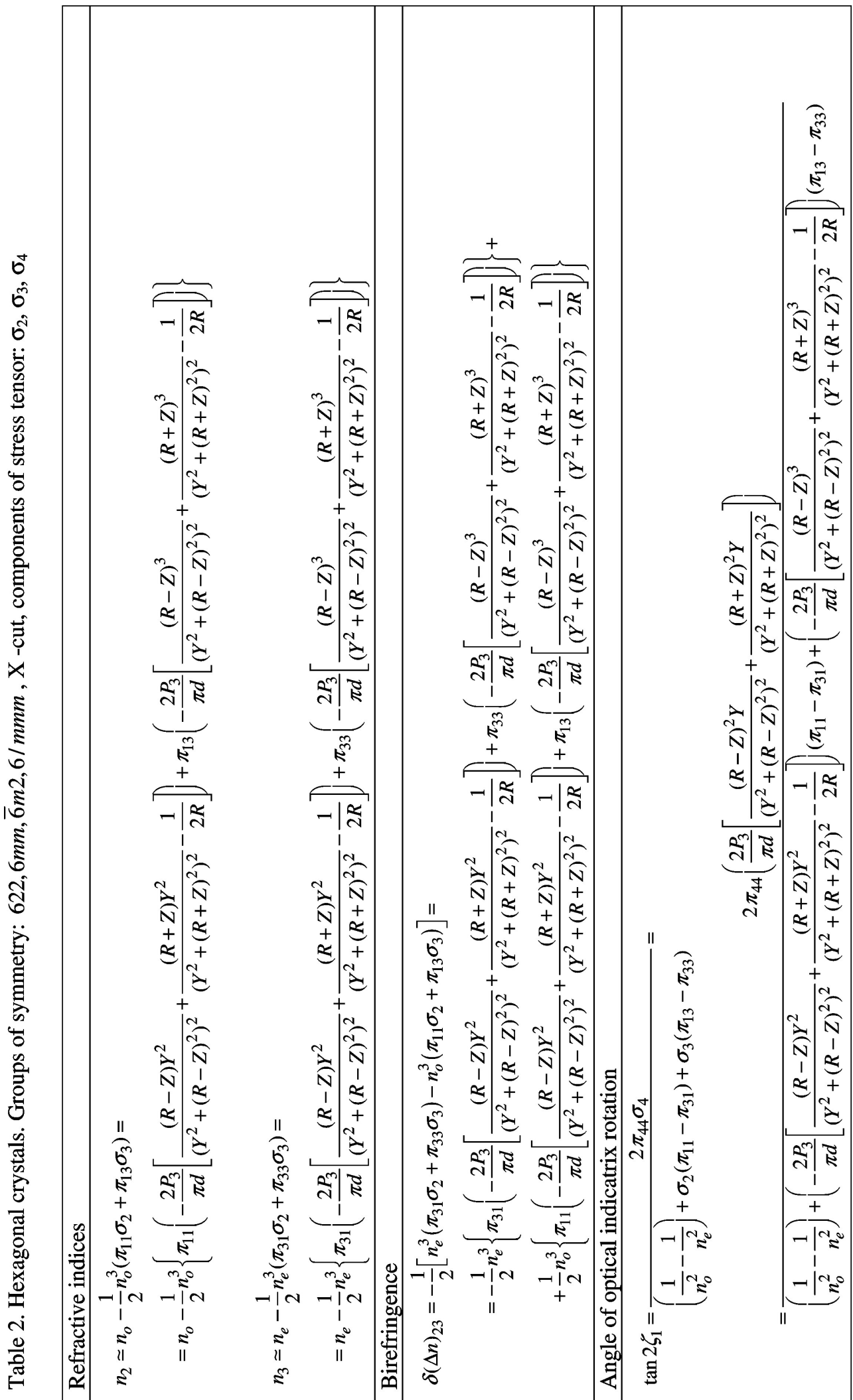




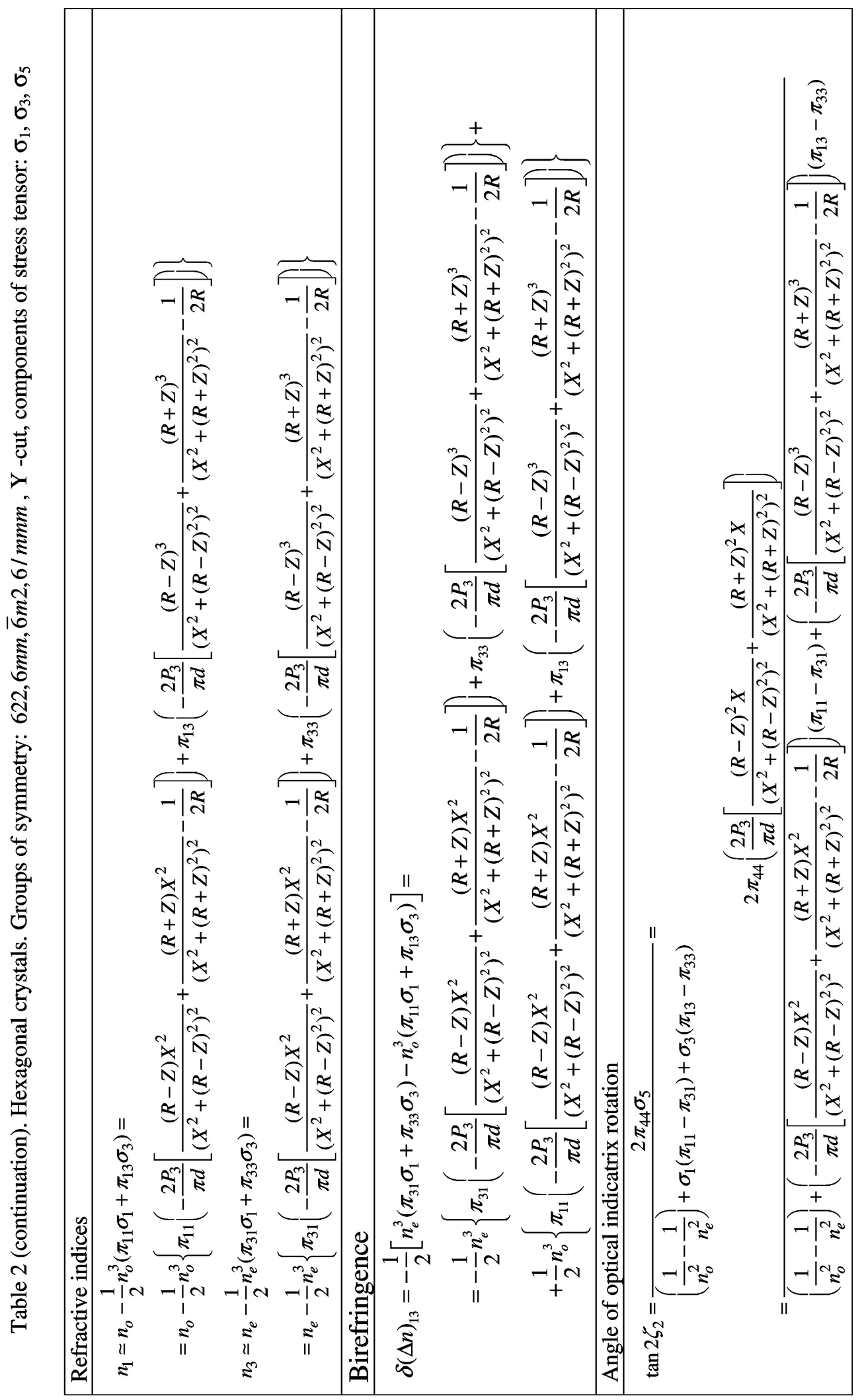




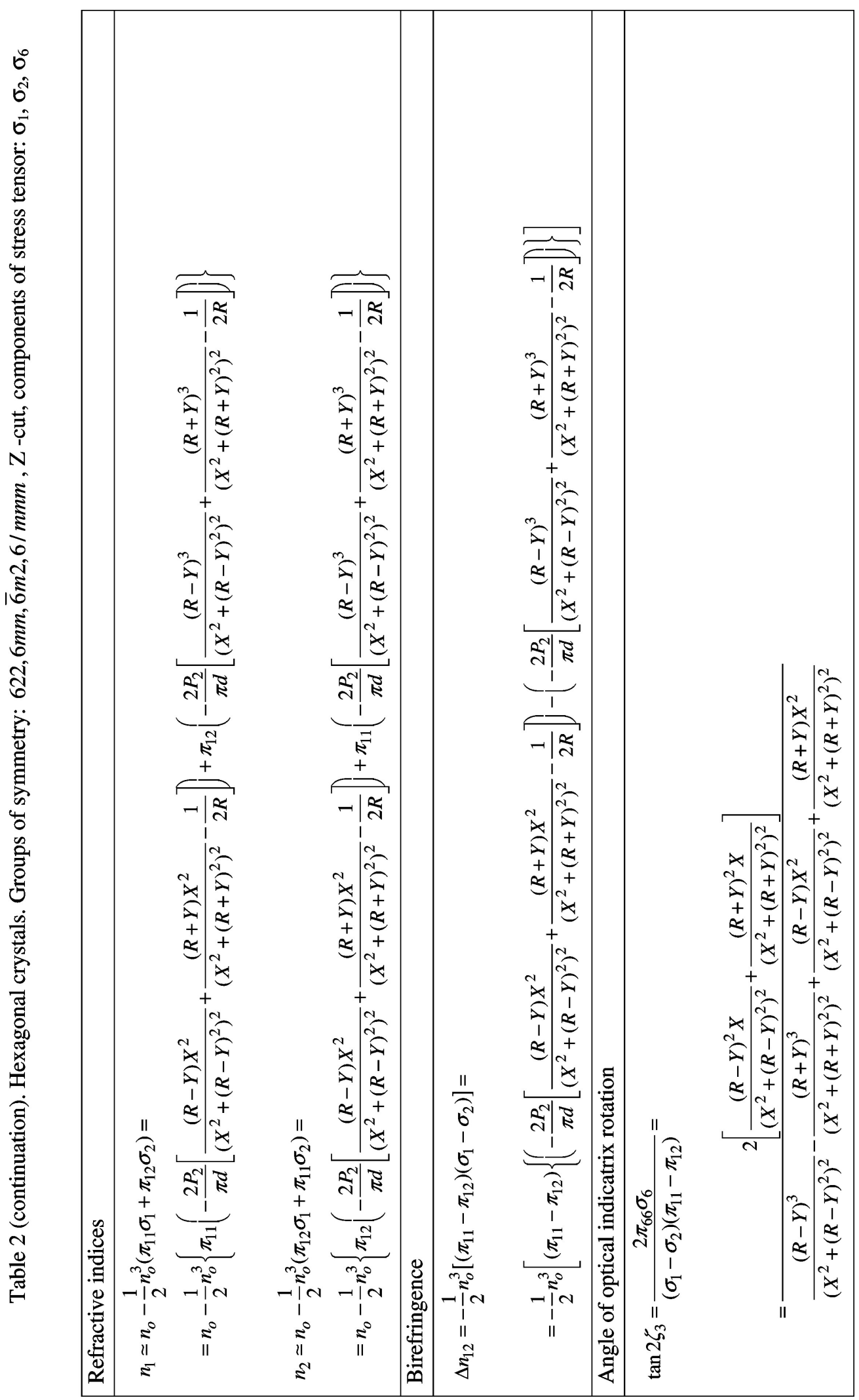




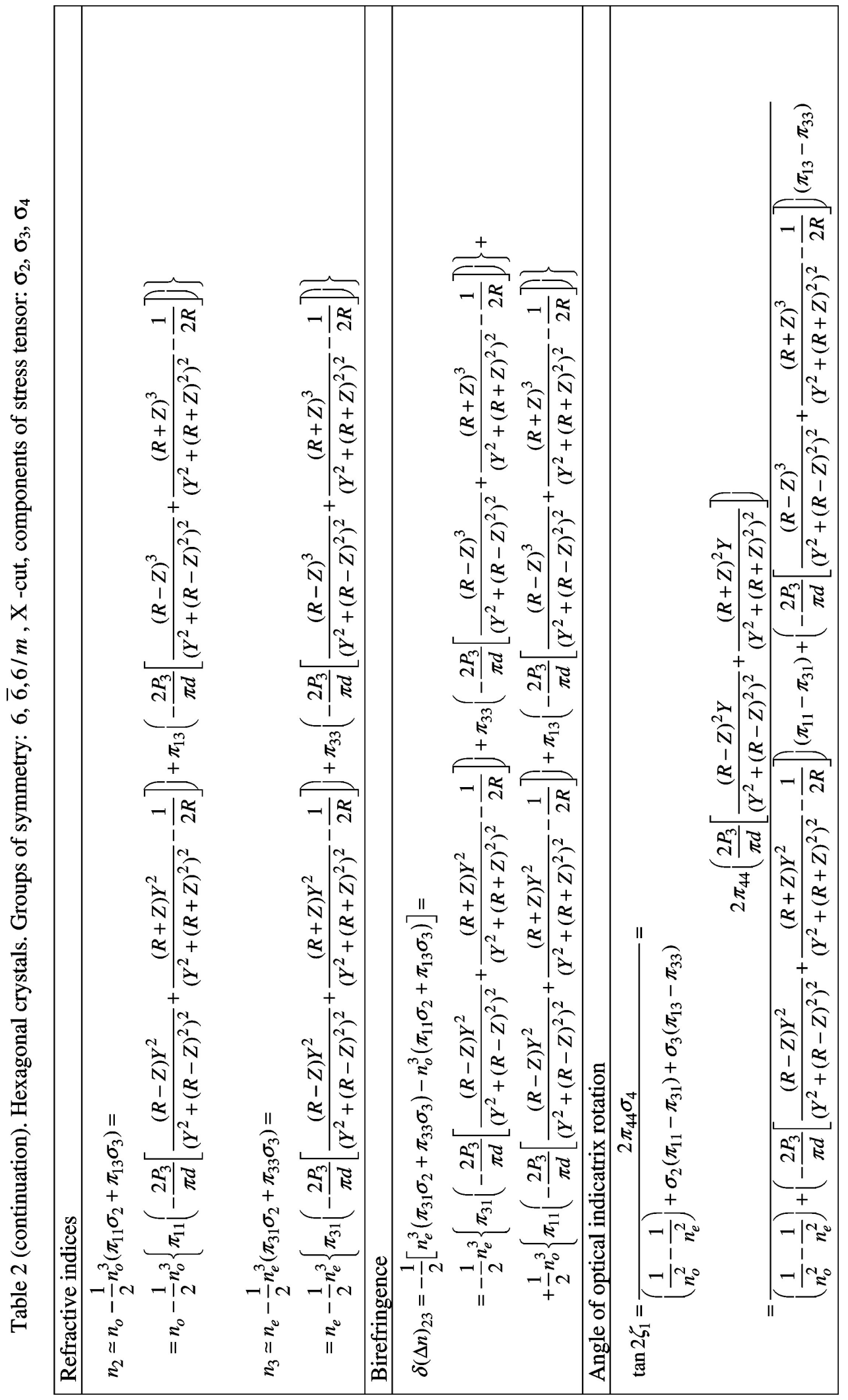




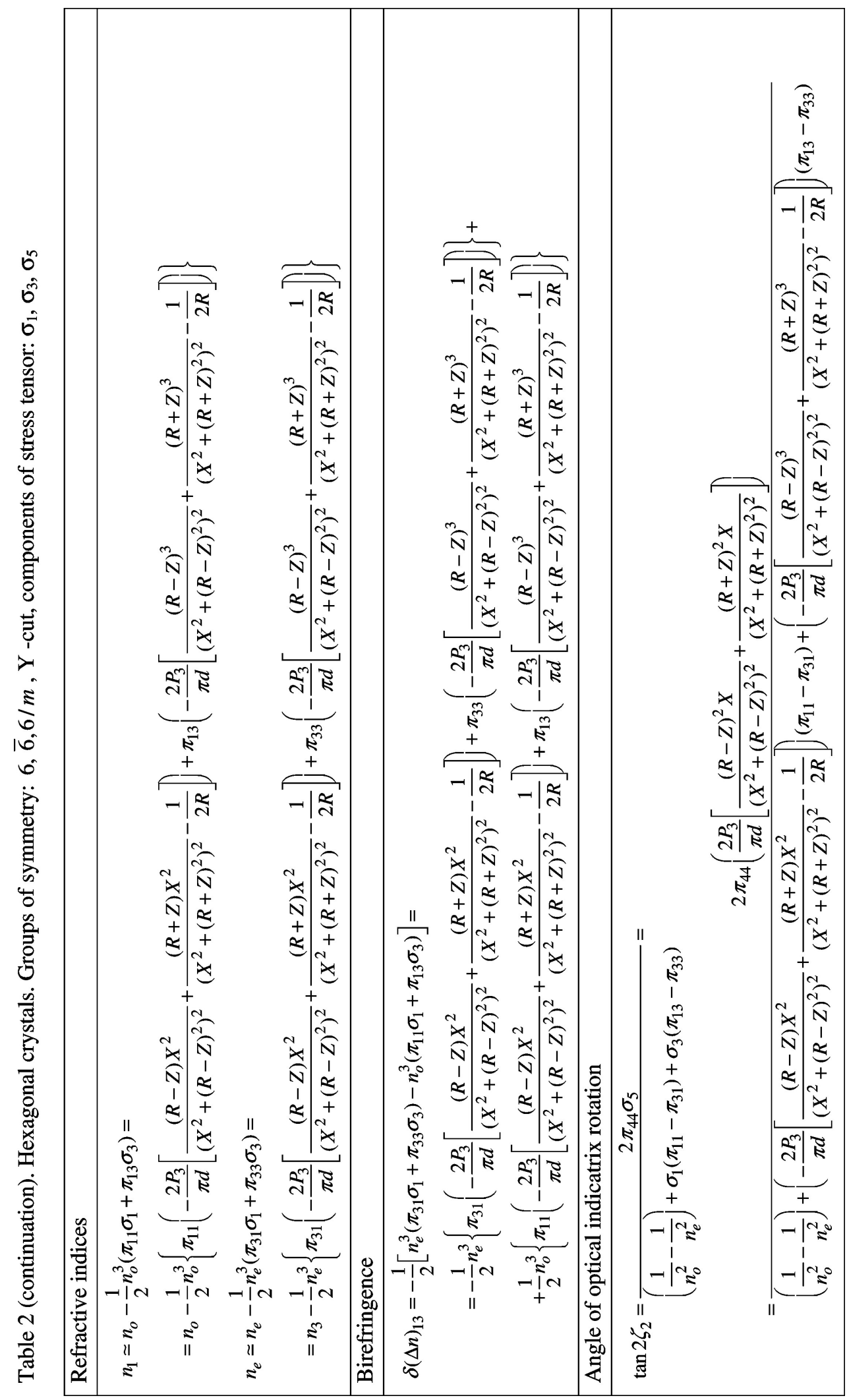




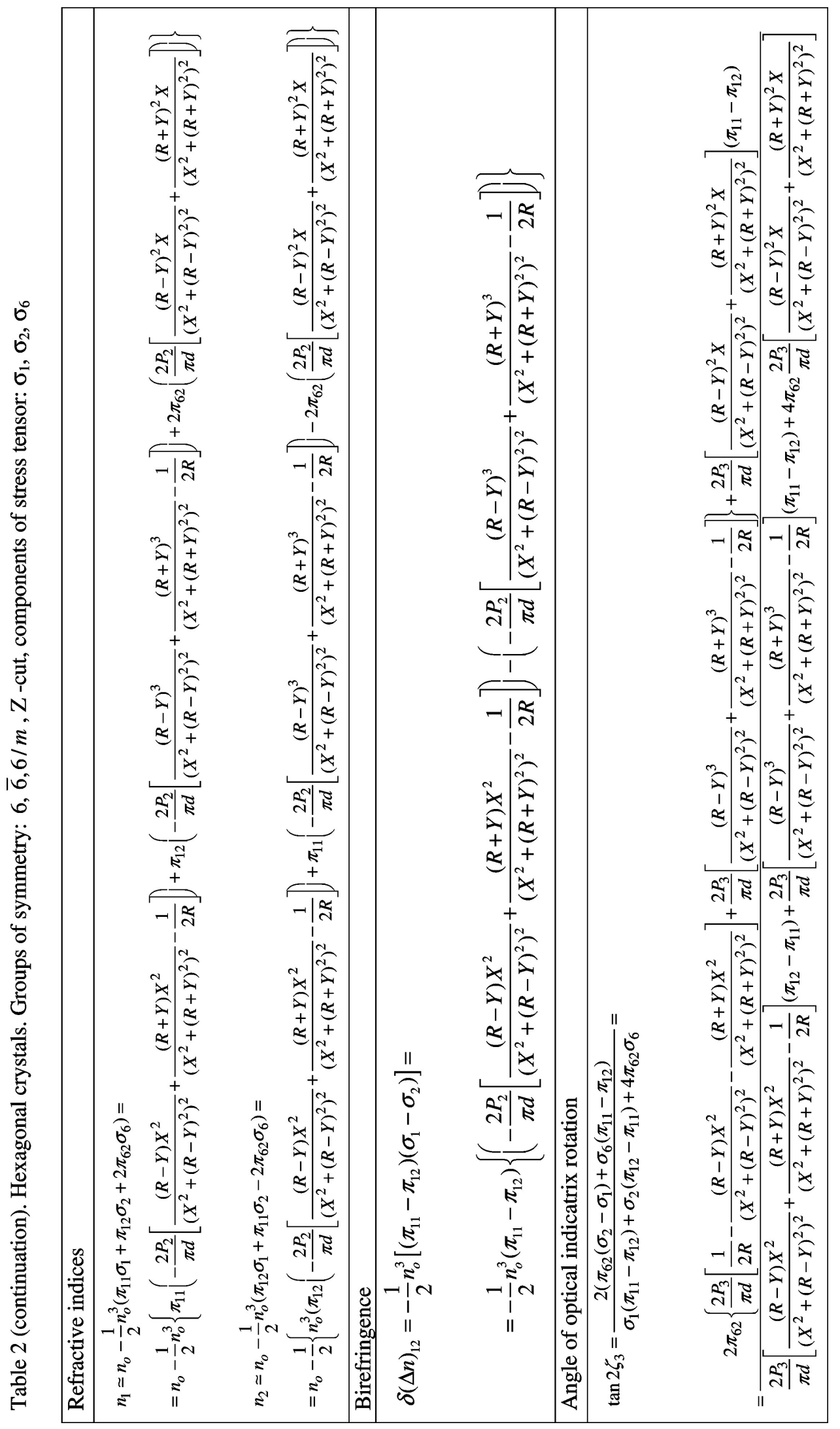

Ukr. J. Phys. Opt. 2012, V13, №2 
The birefringence induced by the force $P_{3}$ for the $Y$-cut disk under the condition $X=0$ becomes

$$
\delta(\Delta n)_{13}=\frac{P_{3}}{\pi d}\left[\frac{1}{2 R}\left(n_{o}^{3} \pi_{11}-n_{e}^{3} \pi_{31}\right)+\left(n_{o}^{3} \pi_{13}-n_{e}^{3} \pi_{33}\right)\left(\frac{1}{2 R}-\frac{2 R}{R^{2}-Z^{2}}\right)\right],
$$

where $n_{e}$ denotes the extraordinary refractive index. This reduces to

$$
\delta(\Delta n)_{13}=\frac{P_{3}}{\pi d} \frac{1}{2 R}\left[\left(n_{o}^{3} \pi_{11}-n_{e}^{3} \pi_{31}\right)-3\left(n_{o}^{3} \pi_{13}-n_{e}^{3} \pi_{33}\right)\right]
$$

at the disk centre $(Z=0)$. This relation includes a complicated combination of four piezooptic coefficients, which can be thus determined. The induced birefringence at $Z=0$ is as follows:

$$
\begin{aligned}
\delta(\Delta n)_{13} & =\frac{P_{3}}{\pi d} \frac{1}{2 R\left(X^{2}+R^{2}\right)^{2}} \times \\
& \times\left[\left(n_{e}^{3} \pi_{31}-n_{o}^{3} \pi_{11}\right)\left(2 R X^{2}-\left(X^{2}+R^{2}\right)^{2}\right)+\left(n_{e}^{3} \pi_{33}-n_{o}^{3} \pi_{13}\right)\left(2 R^{3}-\left(X^{2}+R^{2}\right)^{2}\right)\right] .
\end{aligned}
$$

Neglecting the natural birefringence (assuming the both refractive indices to be close to their mean value, i.e. $n_{o} \simeq n_{e}=\bar{n}$ ), we simplify Eq. (25) to

$$
\begin{aligned}
\delta(\Delta n)_{13} & =\frac{P_{3}}{\pi d} \frac{\bar{n}^{3}}{2 R\left(X^{2}+R^{2}\right)^{2}} \times \\
& \times\left[\left(\pi_{31}-\pi_{11}\right)\left(4 R^{2} X^{2}-\left(X^{2}+R^{2}\right)^{2}\right)+\left(\pi_{33}-\pi_{13}\right)\left(4 R^{4}-\left(X^{2}+R^{2}\right)^{2}\right)\right] .
\end{aligned}
$$

Inserting the piezooptic combination appearing in the square brackets of Eq. (25) into the relation for the optical indicatrix rotation angle,

$$
\tan 2 \zeta_{2}=\frac{8 \pi_{44} R^{3} X}{\left(4 R^{2} X^{2}-\left(X^{2}+R^{2}\right)^{2}\right)\left(\pi_{31}-\pi_{11}\right)+\left(4 R^{4}-\left(X^{2}+R^{2}\right)^{2}\right)\left(\pi_{33}-\pi_{13}\right)},
$$

one can determine the coefficient $\pi_{44}$. This is also true for the point symmetry groups $422,4 \mathrm{~mm}, \overline{4} 2 \mathrm{~m}$ and $4 / \mathrm{mmm}$, for which the relations for the optical indicatrix changes are the same as for the crystals of the groups $622,6 \mathrm{~mm}, \overline{6} \mathrm{~m} 2$ and $6 / \mathrm{mmm}$ (see Table 2), with the only difference that for the tetragonal crystals we have $\pi_{11}-\pi_{12} \neq \pi_{66}$. For the $Z$-cut disk made of tetragonal crystals belonging to the mentioned groups of symmetry, the angle of optical indicatrix rotation at $Y=0$,

$$
\tan 2 \zeta_{3}=\frac{2 \pi_{66} R X}{\left(R^{2}-X^{2}\right)\left(\pi_{11}-\pi_{12}\right)}
$$

enables determining the coefficient $\pi_{66}$ provided that Eq. (20) or Eq. (21) are taken into consideration.

The spatial birefringence distribution along the $Y$ axis for the $Z$-cut disk of crystals that belong to the point symmetry groups $6,6 / \mathrm{m}$ and $\overline{6}$, which are loaded by the force $P_{2}$ may be written as

$$
\Delta n_{12}=-n_{o}^{3}\left(\pi_{11}-\pi_{12}\right) \frac{P_{2}}{\pi d} \frac{2 R}{R^{2}-Y^{2}} .
$$

This facilitates derivation of the difference $\pi_{11}-\pi_{12}=\pi_{66}$. The angle of optical indicatrix rotation under the same conditions, 


$$
\tan 2 \zeta_{3}=\frac{2 \pi_{62}}{\pi_{11}-\pi_{12}},
$$

allows one to obtain the $\pi_{62}$ coefficient whenever the value $\pi_{11}-\pi_{12}$ is already known. Moreover, the relation for the birefringence at $Y=0$,

$$
\Delta n_{12}=2 n_{o}^{3}\left(\pi_{11}-\pi_{12}\right) \frac{P_{2}}{\pi d} \frac{R\left(X^{2}-R^{2}\right)}{\left(X^{2}+R^{2}\right)^{2}},
$$

also allows for determining the above difference of piezooptic coefficients $\pi_{11}-\pi_{12}$, issuing from the birefringence distribution measured in the experiment along the $X$ axis.

Now let us analyse the particular case of the $X$-cut disk prepared from the crystals that belong to the groups $6,6 / \mathrm{m}$ and $\overline{6}$, and the loading force $P_{3}$. Then the spatial distribution of the birefringence increment along the $Y$ axis under the simplifying condition $n_{o} \simeq n_{e}=\bar{n}$ reads as

$$
\begin{aligned}
\delta(\Delta n)_{23}= & \frac{P_{3}}{\pi d} \bar{n}^{3} \frac{1}{2 R\left(Y^{2}+R^{2}\right)^{2}} \times \\
& \times\left[\left(\pi_{31}-\pi_{11}\right)\left(4 R^{2} Y^{2}-\left(Y^{2}+R^{2}\right)^{2}\right)+\left(\pi_{33}-\pi_{13}\right)\left(4 R^{4}-\left(Y^{2}+R^{2}\right)^{2}\right)\right]
\end{aligned}
$$

After measuring additionally the distribution of the optical indicatrix rotation angle along the $Y$ axis,

$$
\tan 2 \zeta_{1}=\frac{8 \pi_{44} R^{3} Y}{\left(4 R^{2} Y^{2}-\left(Y^{2}+R^{2}\right)^{2}\right)\left(\pi_{31}-\pi_{11}\right)+\left(4 R^{4}-\left(Y^{2}+R^{2}\right)^{2}\right)\left(\pi_{33}-\pi_{13}\right)},
$$

one can evaluate the coefficient $\pi_{44}$.

\subsection{Tetragonal crystals}

The coefficient $\pi_{44}$ for the tetragonal crystals can be obtained in the same way as that for the hexagonal ones. However, the appropriate relations for the Z-cut disk in case of the groups of symmetry $4,4 / \mathrm{m}$ and $\overline{4}$ differ from those valid for the hexagonal crystals (see Table 3 ). In particular, the birefringence in the case of $X=0$ acquires the following form:

$$
\delta(\Delta n)_{12}=\frac{P_{2}}{\pi d} n_{o}^{3}\left(\pi_{11}-\pi_{12}\right) \frac{2 R}{R^{2}-Y^{2}} .
$$

Basing on the experimental dependence of the birefringence increment on the $Y$ coordinate, this relation enables evaluating the piezoptic difference $\pi_{11}-\pi_{12}$. Then the coefficient $\pi_{61}$ can be determined from the relation for the optical indicatrix rotation at $X=0$,

$$
\tan 2 \zeta_{3}=\frac{2 \pi_{61}}{\pi_{11}-\pi_{12}}
$$

Finally, using the theoretical relations for the birefringence at $Y=0$,

$$
\Delta n_{12}=n_{o}^{3} \frac{P_{2}}{\pi d} \frac{2 R}{\left(X^{2}+R^{2}\right)^{2}}\left(\left(\pi_{11}-\pi_{12}\right)\left(X^{2}-R^{2}\right)-2 \pi_{16} R X\right),
$$

and for the angle of optical indicatrix rotation at $Y=0$,

$$
\tan 2 \zeta_{3}=\frac{2 \pi_{61}\left(X^{2}-R^{2}\right)-2 \pi_{66} R X}{\left(X^{2}-R^{2}\right)\left(\pi_{11}-\pi_{12}\right)-2 \pi_{16} R X},
$$

one can find the coefficients $\pi_{66}$ and $\pi_{16}$, provided that the coefficient $\pi_{61}$ is already known. 


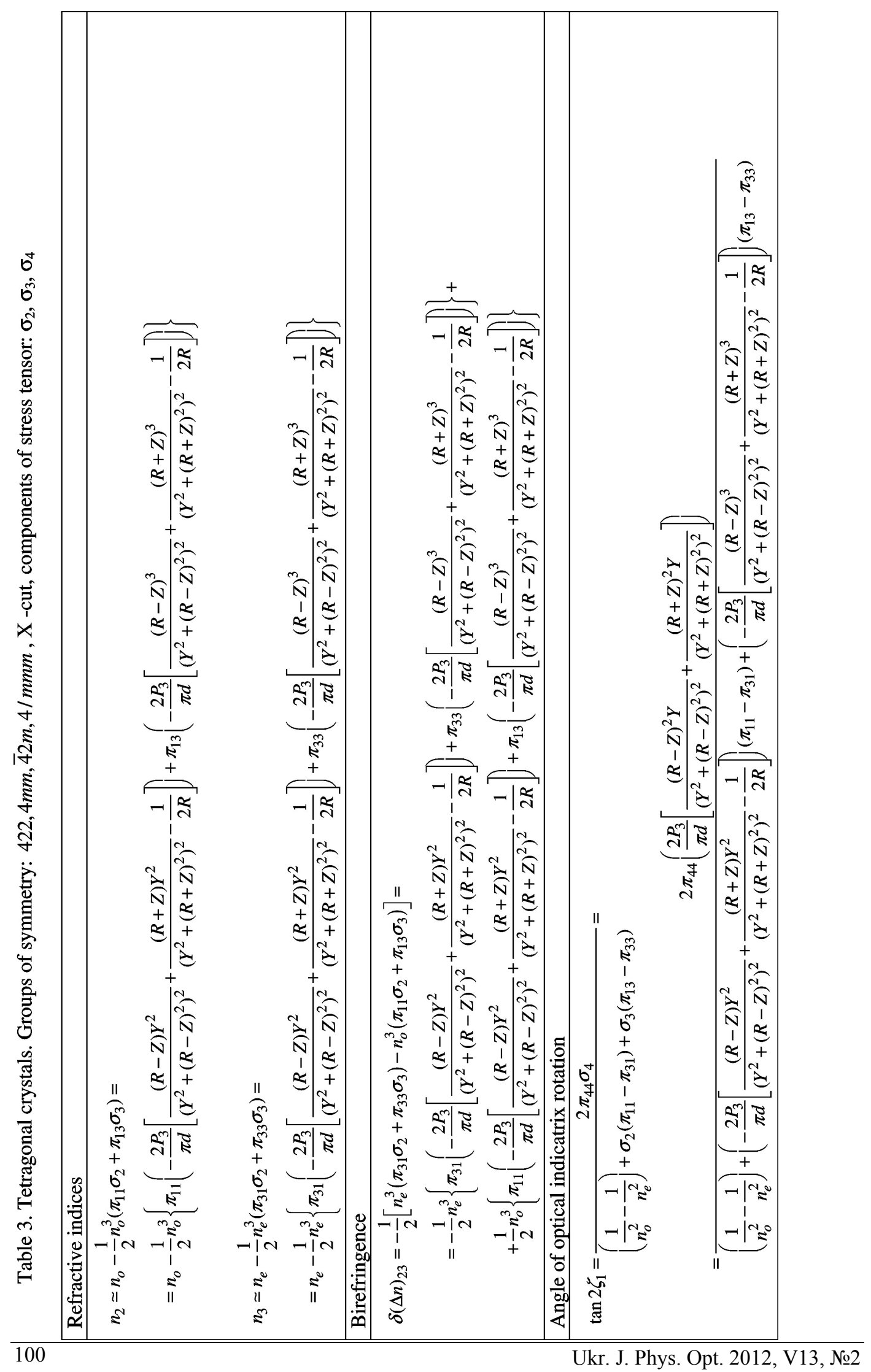




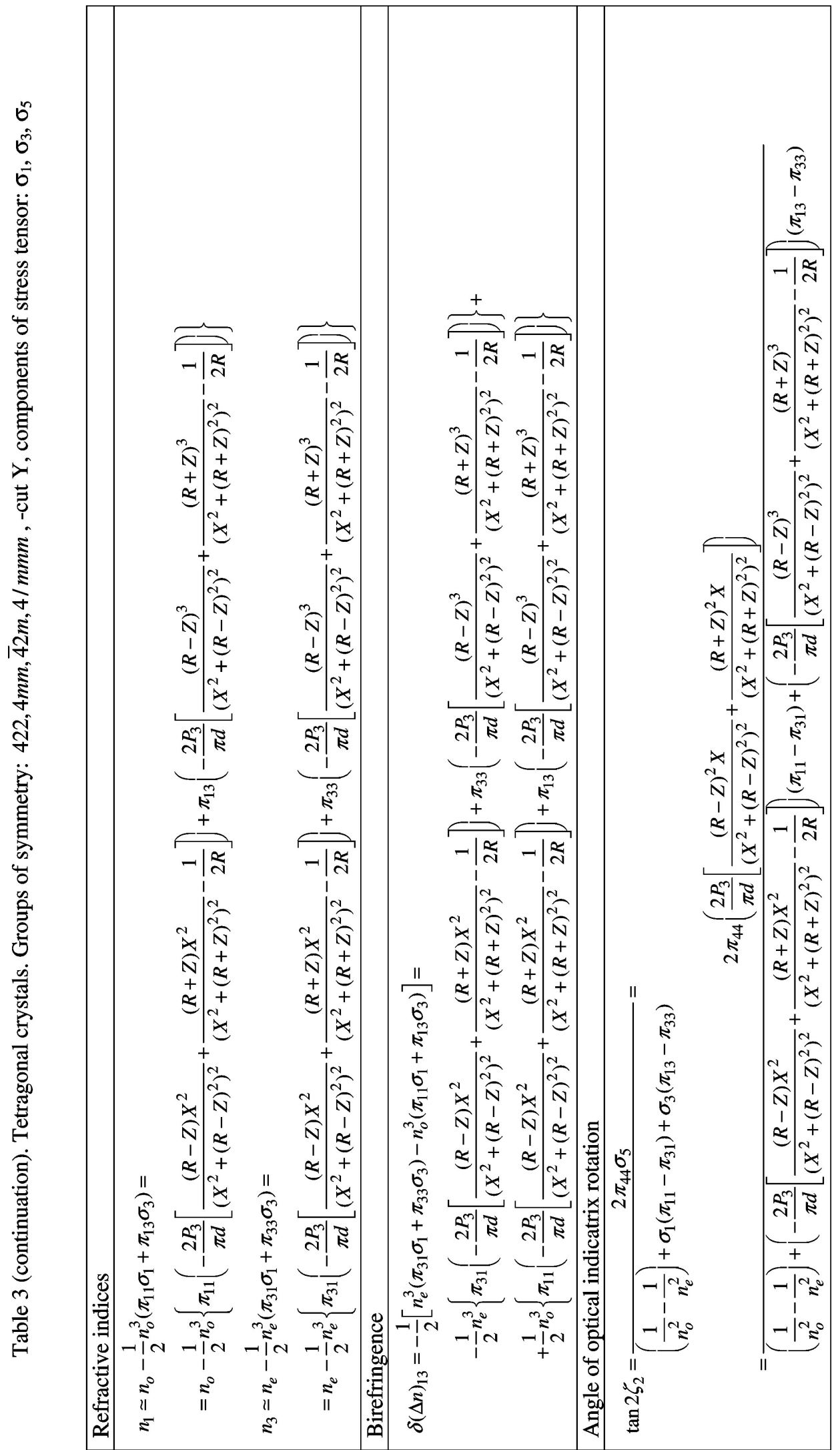

Ukr. J. Phys. Opt. 2012, V13, №2 


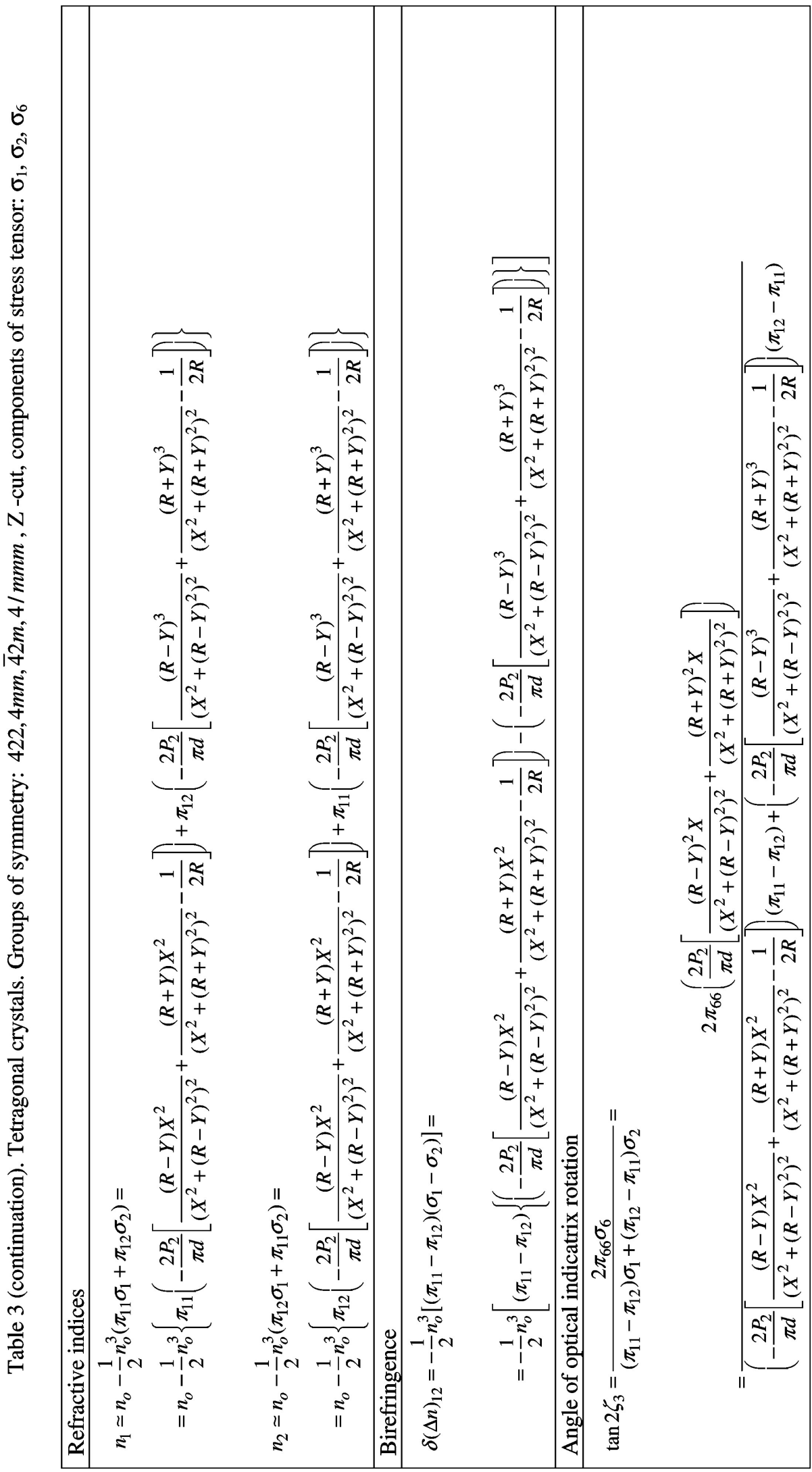




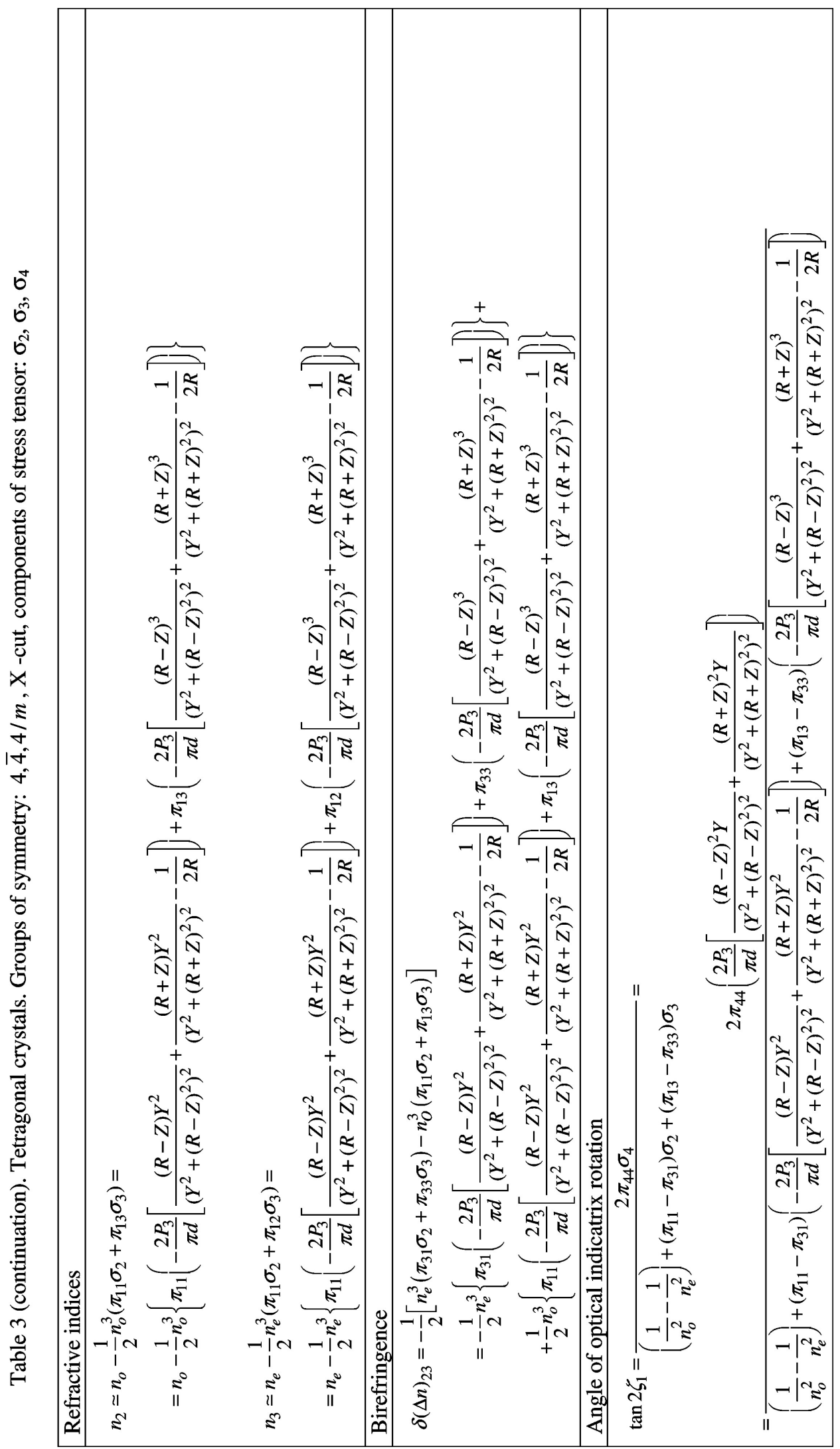

Ukr. J. Phys. Opt. 2012, V13, №2 


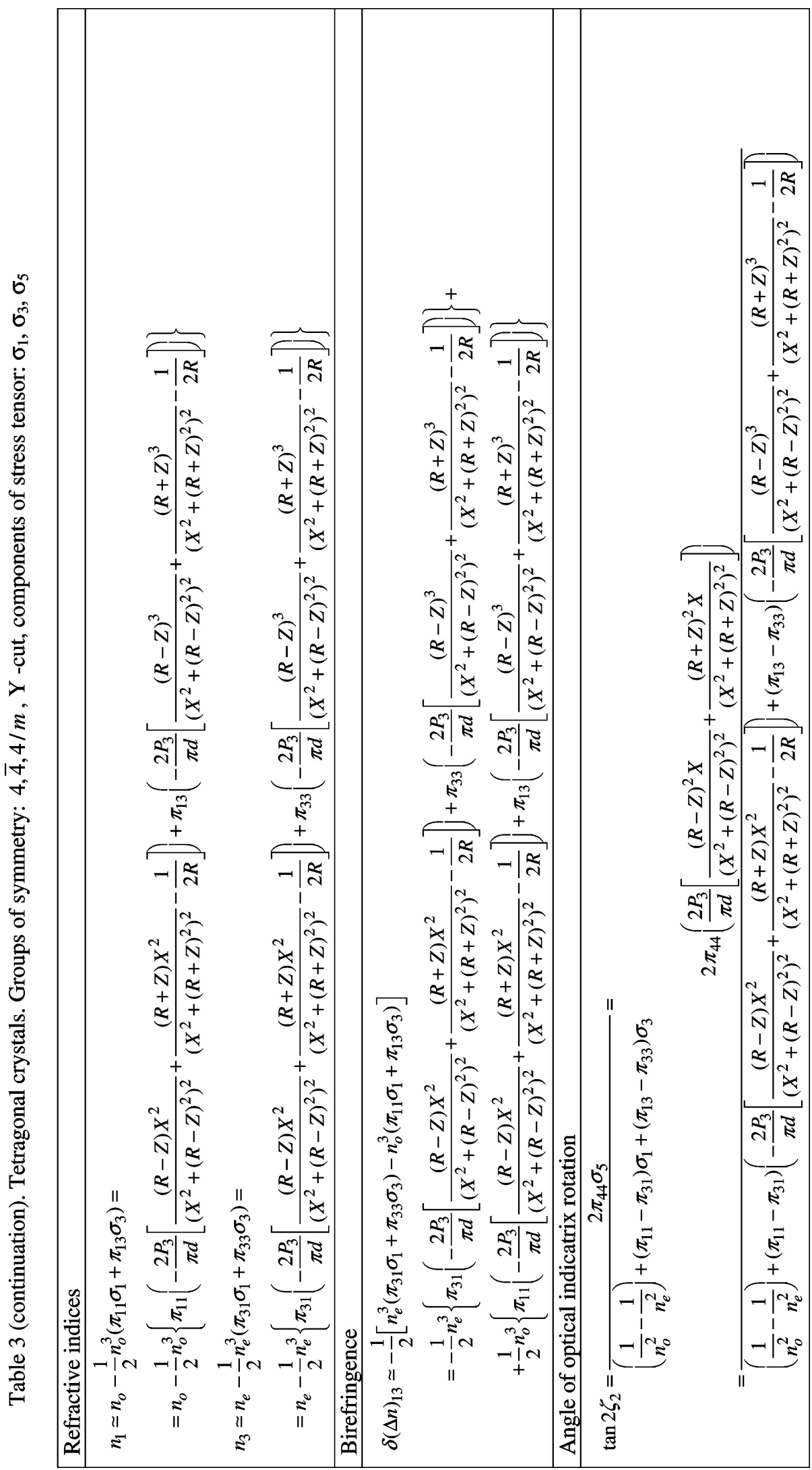




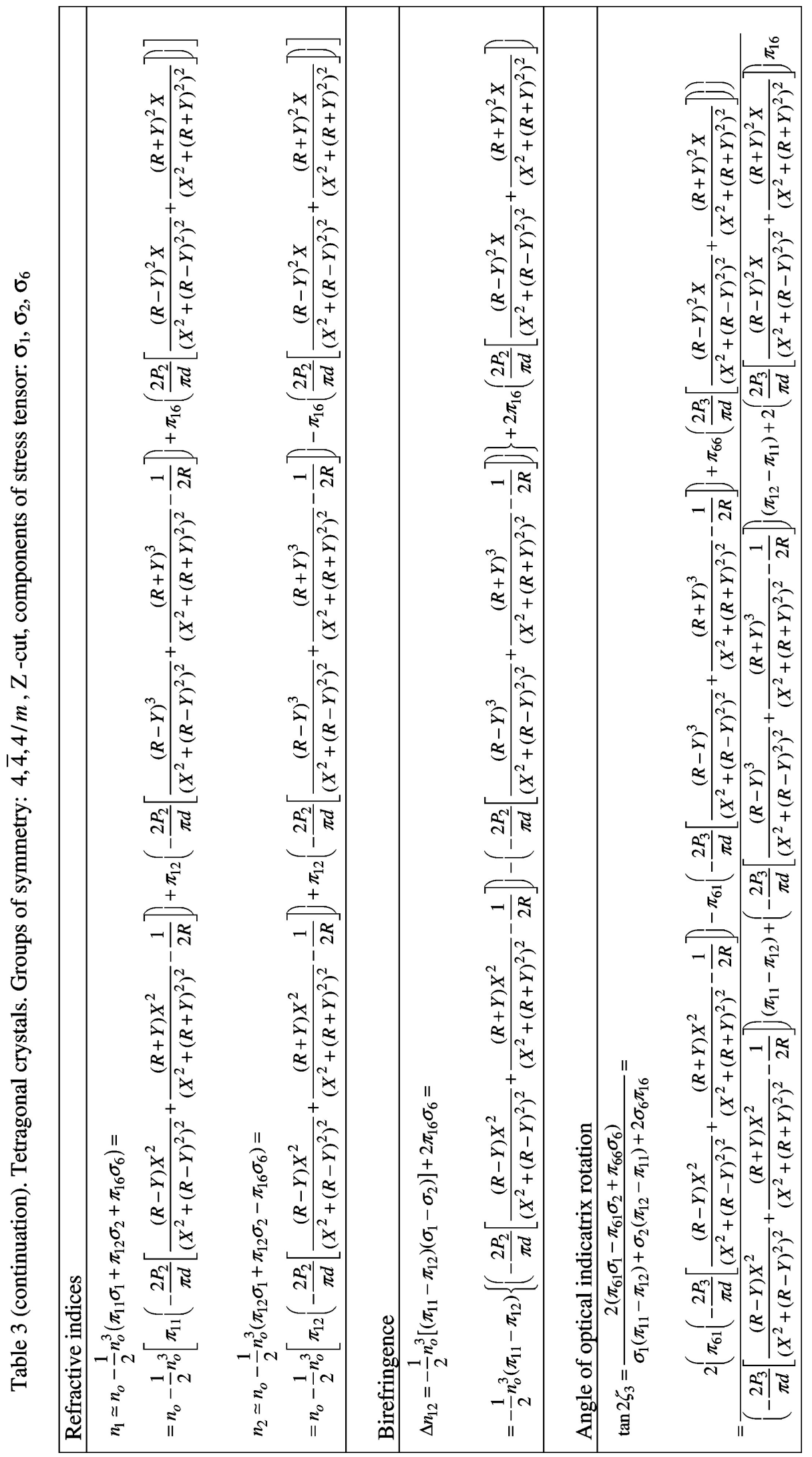

Ukr. J. Phys. Opt. 2012, V13, №2 


\subsection{Trigonal crystals}

Phenomenological relations for the changes happening in the optical indicatrix parameters for the crystals of point symmetry groups $32,3 \mathrm{~m}$ and $\overline{3} \mathrm{~m}$ have been derived in our recent work [10]. As follows from the results [10], the analysis presented in the beginning of Section 2 and Table 4, one can easily determine the combined difference $\pi_{66}=\pi_{11}-\pi_{12}$ for these crystals. The procedure of evaluation of the coefficients $\pi_{44}$ and $\pi_{41}$ is described in the work [10], too.

Let us discuss the crystals belonging to the symmetry groups 3 and $\overline{3}$. The birefringence for the disk perpendicular to the $Z$ axis, the loading force $P_{2}$ and the experimental geometry $X=0$ is given by

$$
\Delta n_{12}=-\frac{P_{2}}{\pi d} n_{o}^{3}\left(\pi_{11}-\pi_{12}\right)\left[\frac{2 R}{R^{2}-Y^{2}}-\frac{1}{2 R}\right] .
$$

Using this relation and the induced birefringence distribution along the $Y$ axis studied in the experiment, one can find the piezooptic difference $\pi_{66}=\pi_{11}-\pi_{12}$.

For the $X$-cut disk, under the condition $Z=0$ and the assumption $n_{o} \simeq n_{e}=\bar{n}$, the relation for the induced birefringence becomes

$$
\begin{aligned}
\delta(\Delta n)_{23}= & \frac{P_{3}}{\pi d} \bar{n}^{3} \frac{1}{2 R\left(Y^{2}+(R)^{2}\right)^{2}} \times \\
& \times\left[\left(\pi_{31}-\pi_{11}\right)\left[4 R^{2} Y^{2}-\left(Y^{2}+R^{2}\right)^{2}\right]+\left(\pi_{33}-\pi_{13}\right)\left[4 R^{4}-\left(Y^{2}+R^{2}\right)^{2}\right]-4 R^{3} Y \pi_{14}\right]
\end{aligned}
$$

Basing on the formula for the optical indicatrix rotation obtained under the condition $Z=0$,

$$
\tan 2 \zeta_{1}=\frac{2\left\{4 R^{3} Y \pi_{44}+\pi_{41}\left[4 R^{2} Y^{2}-\left(Y^{2}+R^{2}\right)^{2}\right]\right\}}{\left[4 R^{2} Y^{2}-\left(Y^{2}+R^{2}\right)^{2}\right]\left(\pi_{31}-\pi_{11}\right)+\left[4 R^{4}-\left(Y^{2}+R^{2}\right)^{2}\right]\left(\pi_{33}-\pi_{13}\right)-4 R^{3} Y \pi_{14}},
$$

and inserting the known piezooptic combination from Eq. (39) into denominator of Eq. (40), one can derive the combination of coefficients $\pi_{44}$ and $\pi_{41}$. At the same time, Eq. (40) for the case of $Y=0$ may be rewritten to the form

$$
\tan 2 \zeta_{1}=\frac{2 \pi_{41}}{\left(\pi_{31}-\pi_{11}\right)-3\left(\pi_{33}-\pi_{13}\right)},
$$

thus enabling independent evaluation of the coefficients $\pi_{41}$ and $\pi_{44}$.

\subsection{Orthorhombic crystals}

For the crystals that belong to the orthorhombic groups of symmetry (see Table 5), one can easily determine the coefficients $\pi_{44}, \pi_{55}$ and $\pi_{66}$. For example, using the $X$-cut disk and assuming $n_{2} \simeq n_{3}=\bar{n}$ under the condition $Z=0$, one can arrive at the following relations for the birefringence increment and the angle of optical indicatrix rotation:

$$
\begin{aligned}
\delta(\Delta n)_{23}= & \bar{n}^{3} \frac{P_{3}}{\pi d} \frac{1}{2 R\left(Y^{2}+(R)^{2}\right)^{2}} \times \\
& \times\left[\left(\pi_{22}-\pi_{32}\right)\left[4 R^{2} Y^{2}-\left(Y^{2}+R^{2}\right)^{2}\right]+\left(\pi_{23}-\pi_{33}\right)\left[4 R^{4}-\left(Y^{2}+R^{2}\right)^{2}\right]\right], \\
\tan 2 \zeta_{1}= & -\frac{8 R^{2} Y^{2} \pi_{44}}{\left[4 R^{2} Y^{2}-\left(Y^{2}+R^{2}\right)^{2}\right]\left(\pi_{22}-\pi_{32}\right)+\left[4 R^{4}-\left(Y^{2}+R^{2}\right)^{2}\right]\left(\pi_{23}-\pi_{33}\right)} .
\end{aligned}
$$




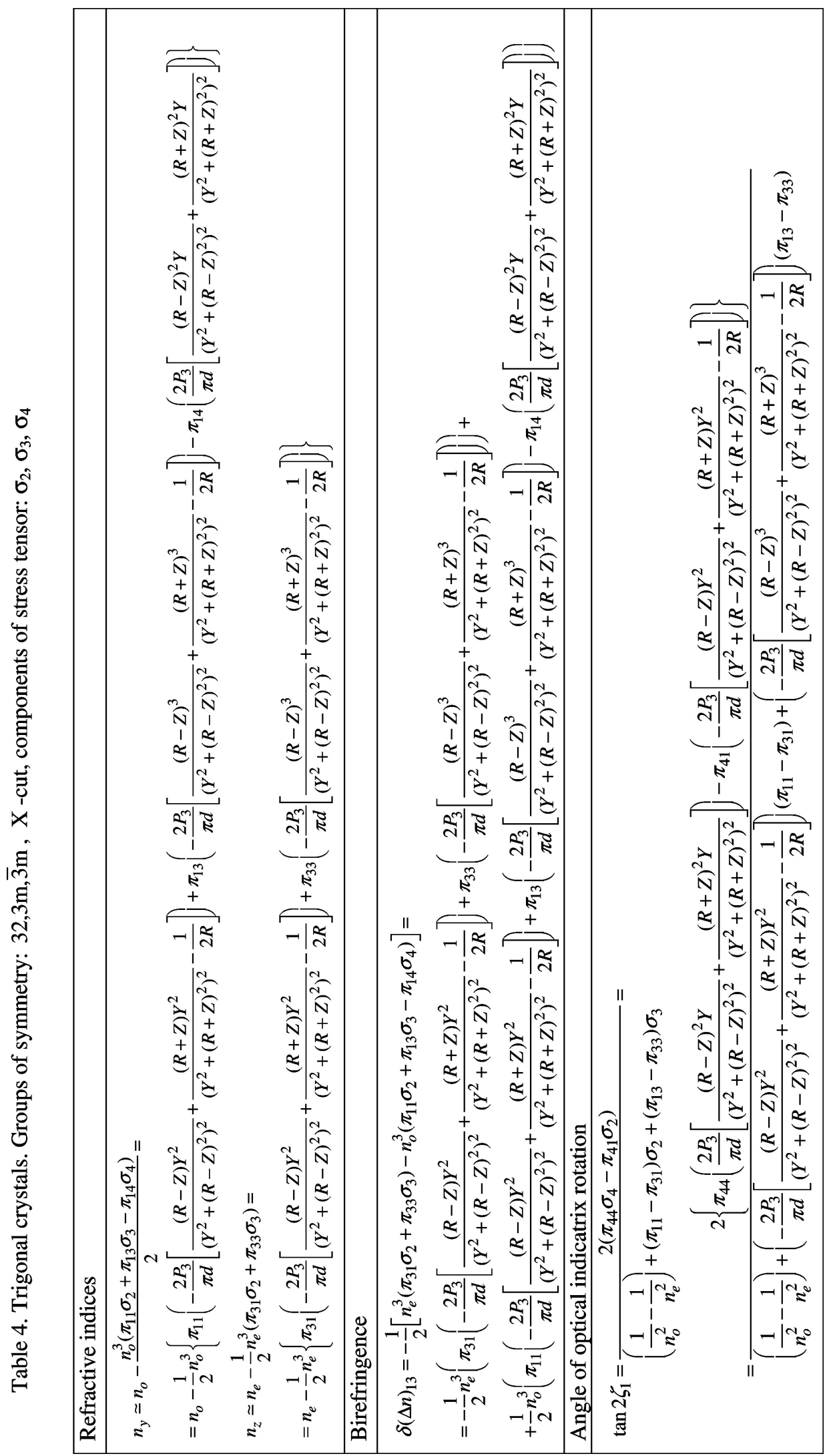

Ukr. J. Phys. Opt. 2012, V13, №2 


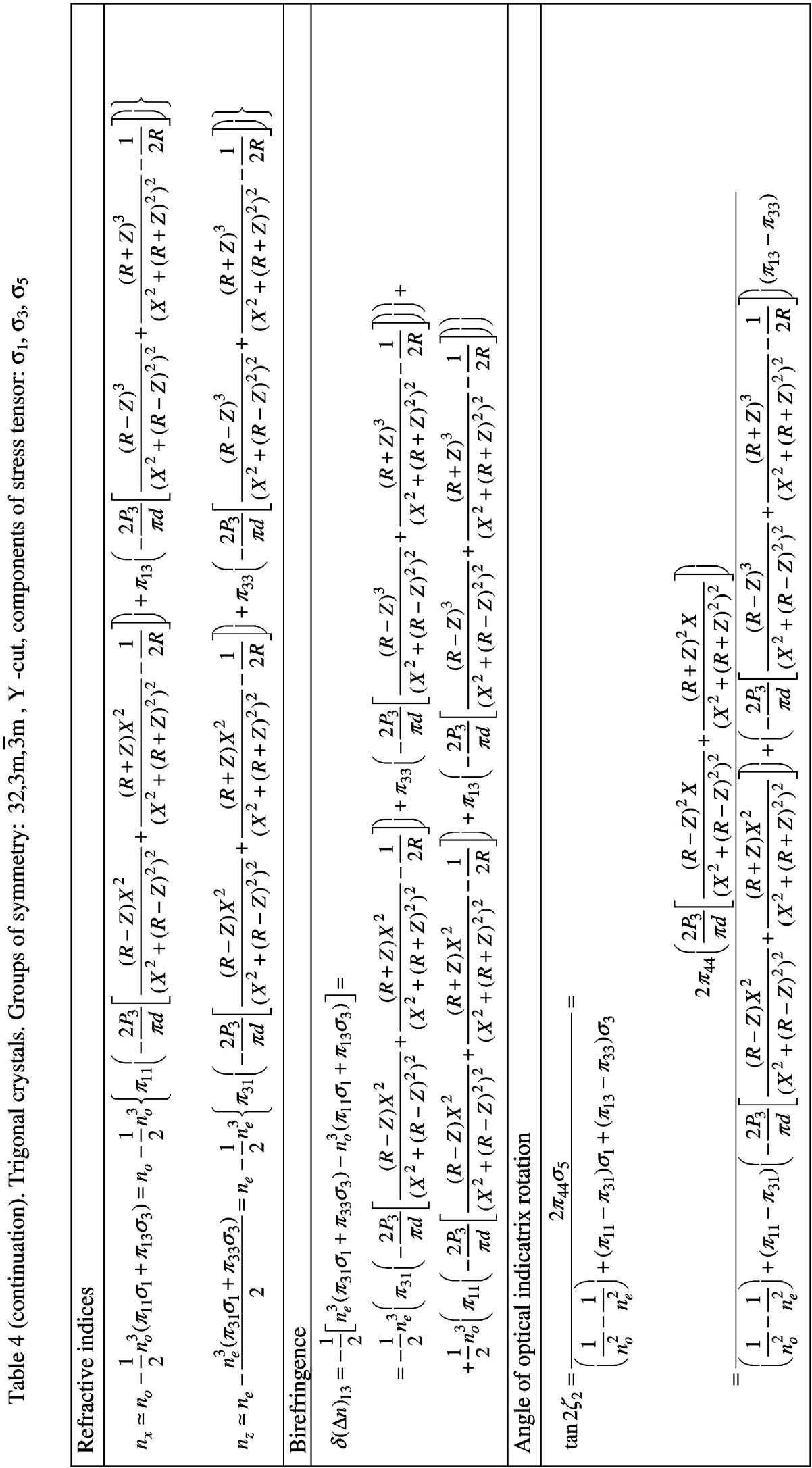




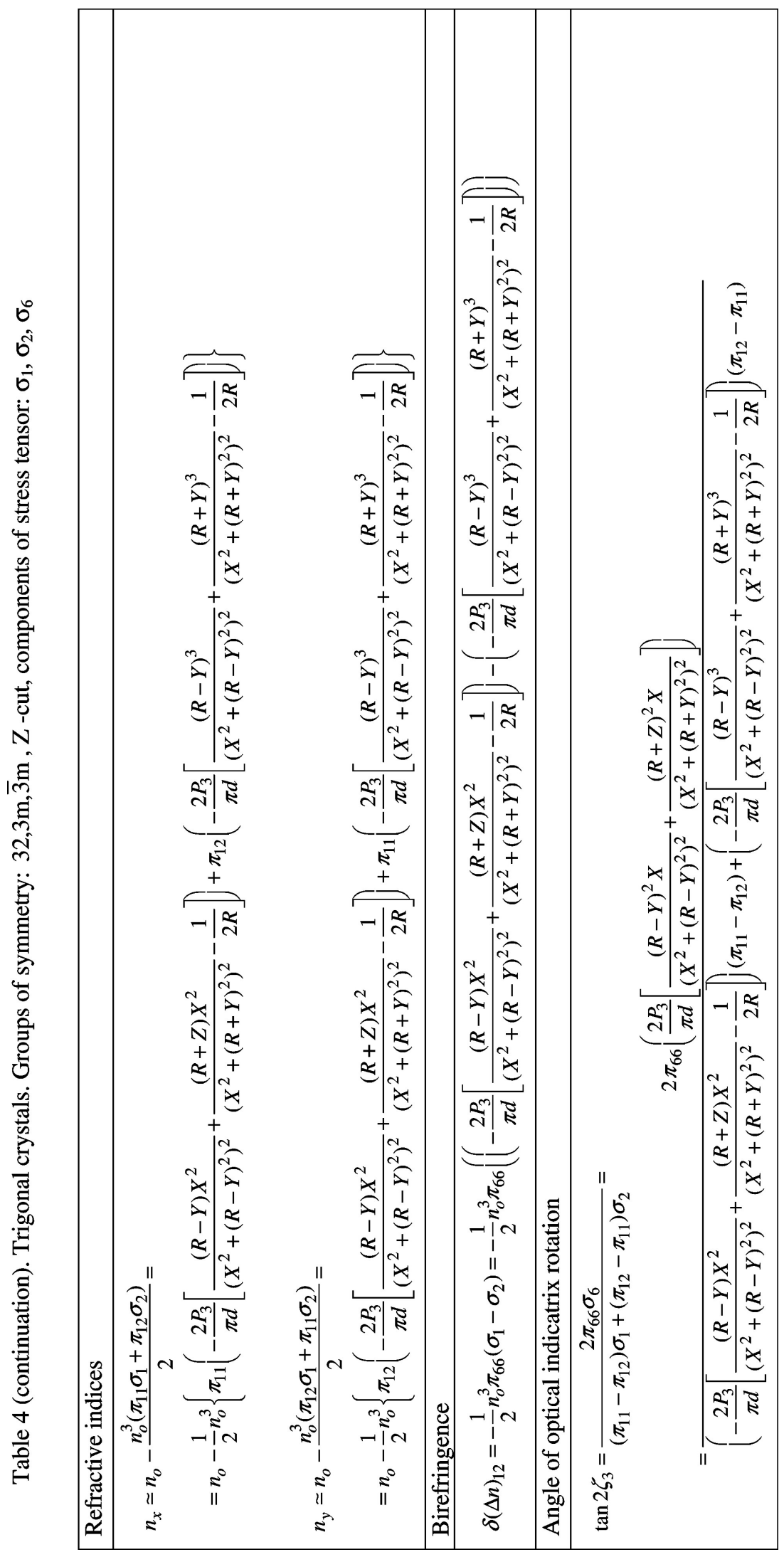

Ukr. J. Phys. Opt. 2012, V13, №2 


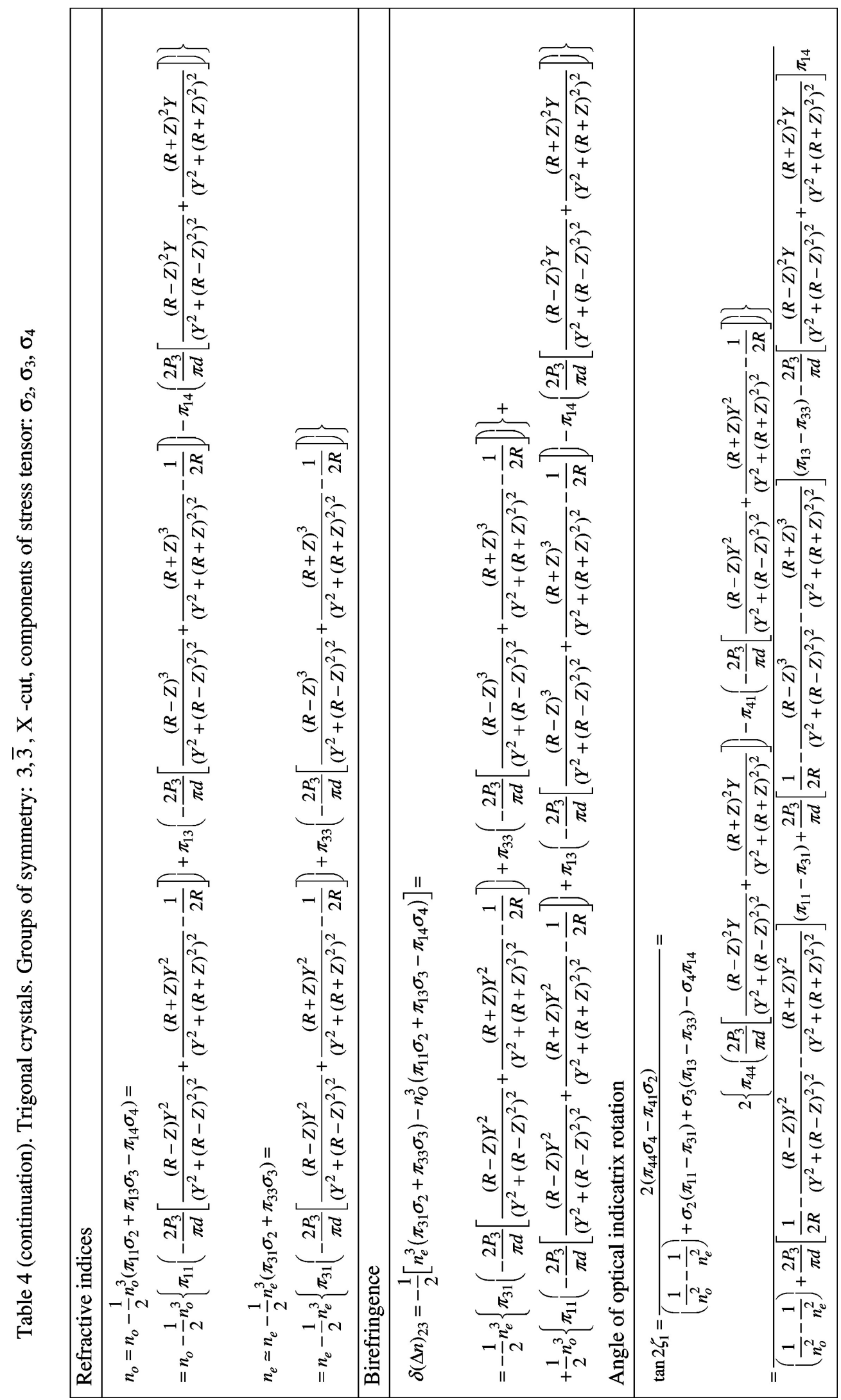




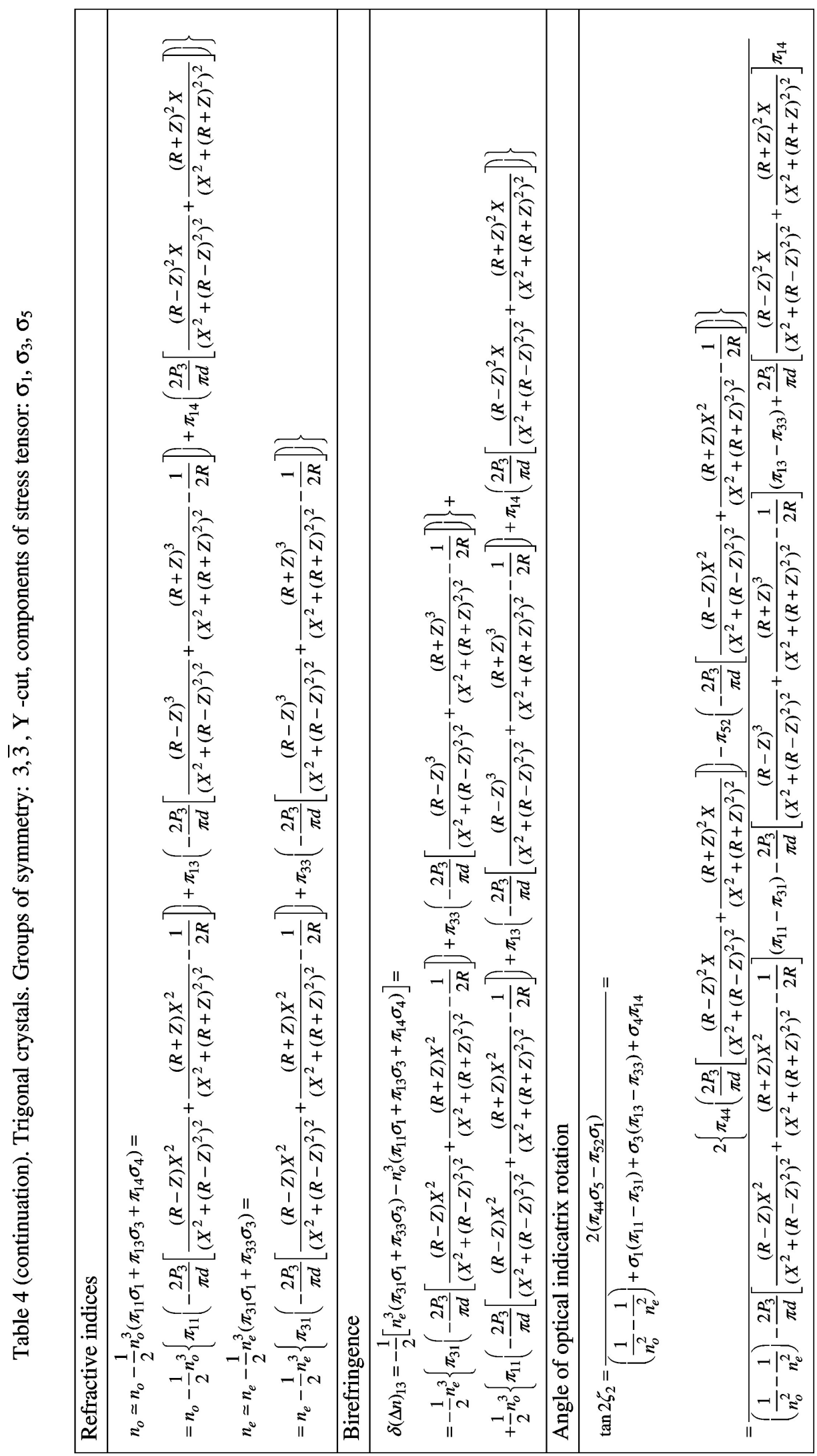

Ukr. J. Phys. Opt. 2012, V13, №2 


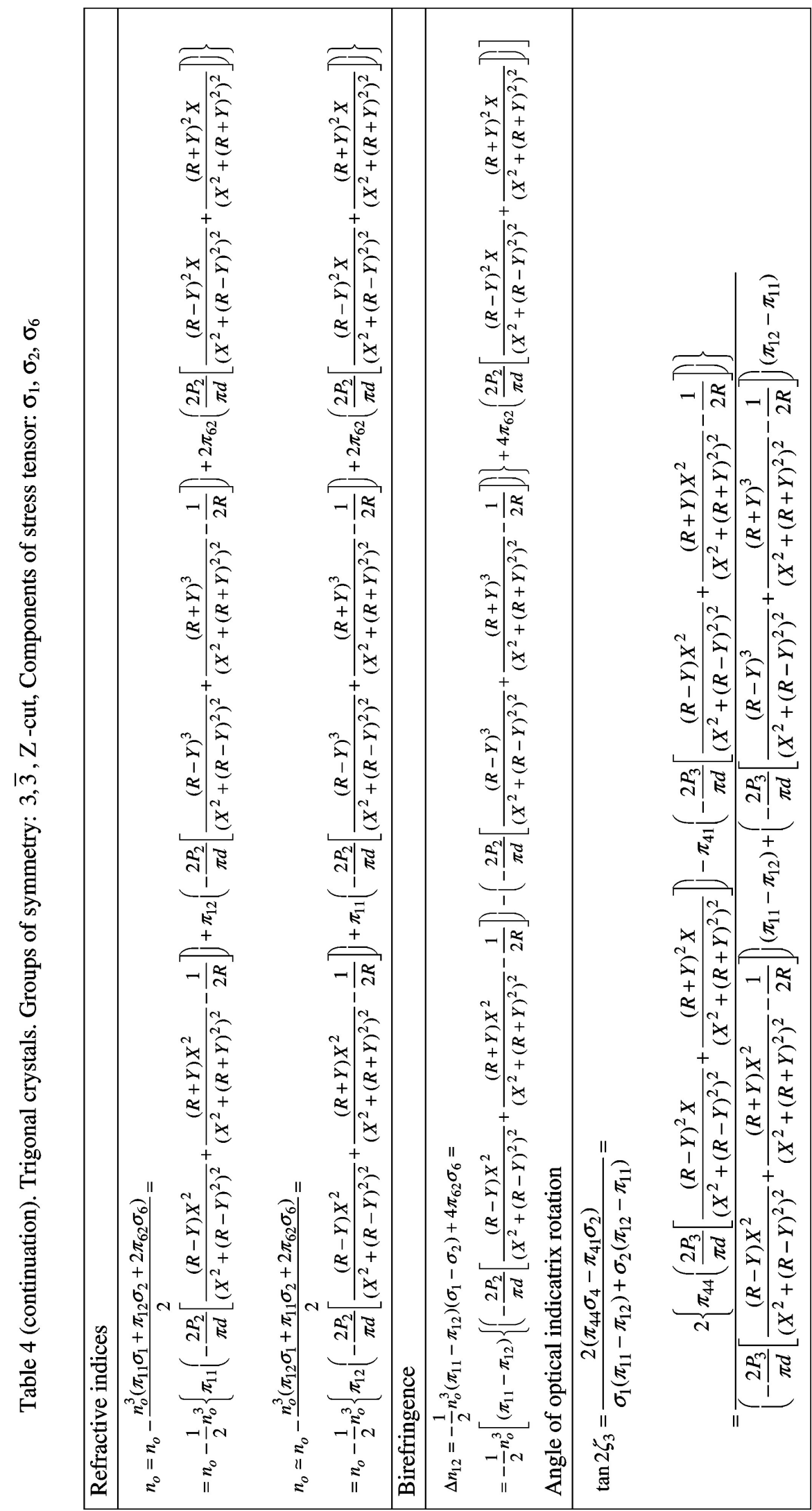




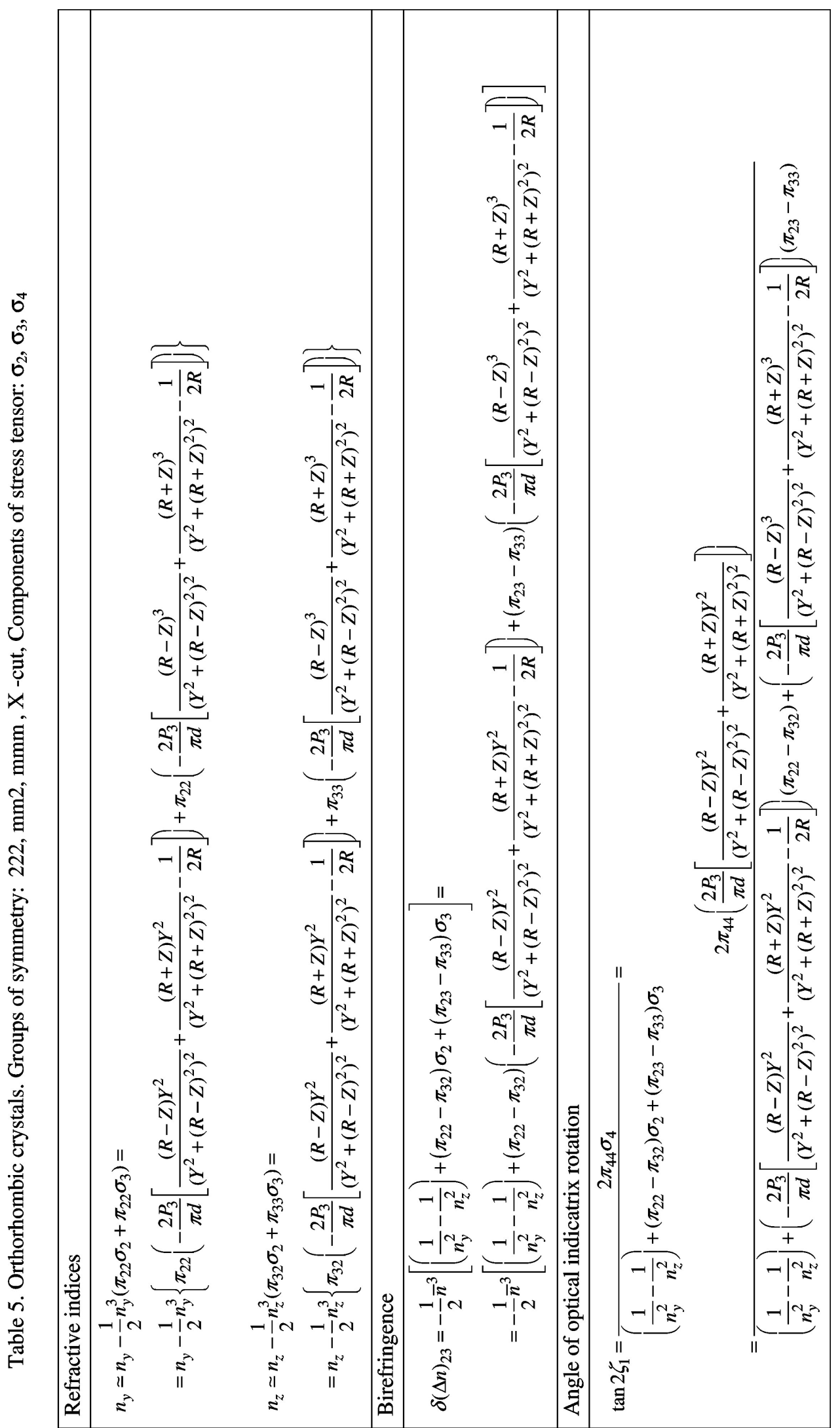

Ukr. J. Phys. Opt. 2012, V13, №2 


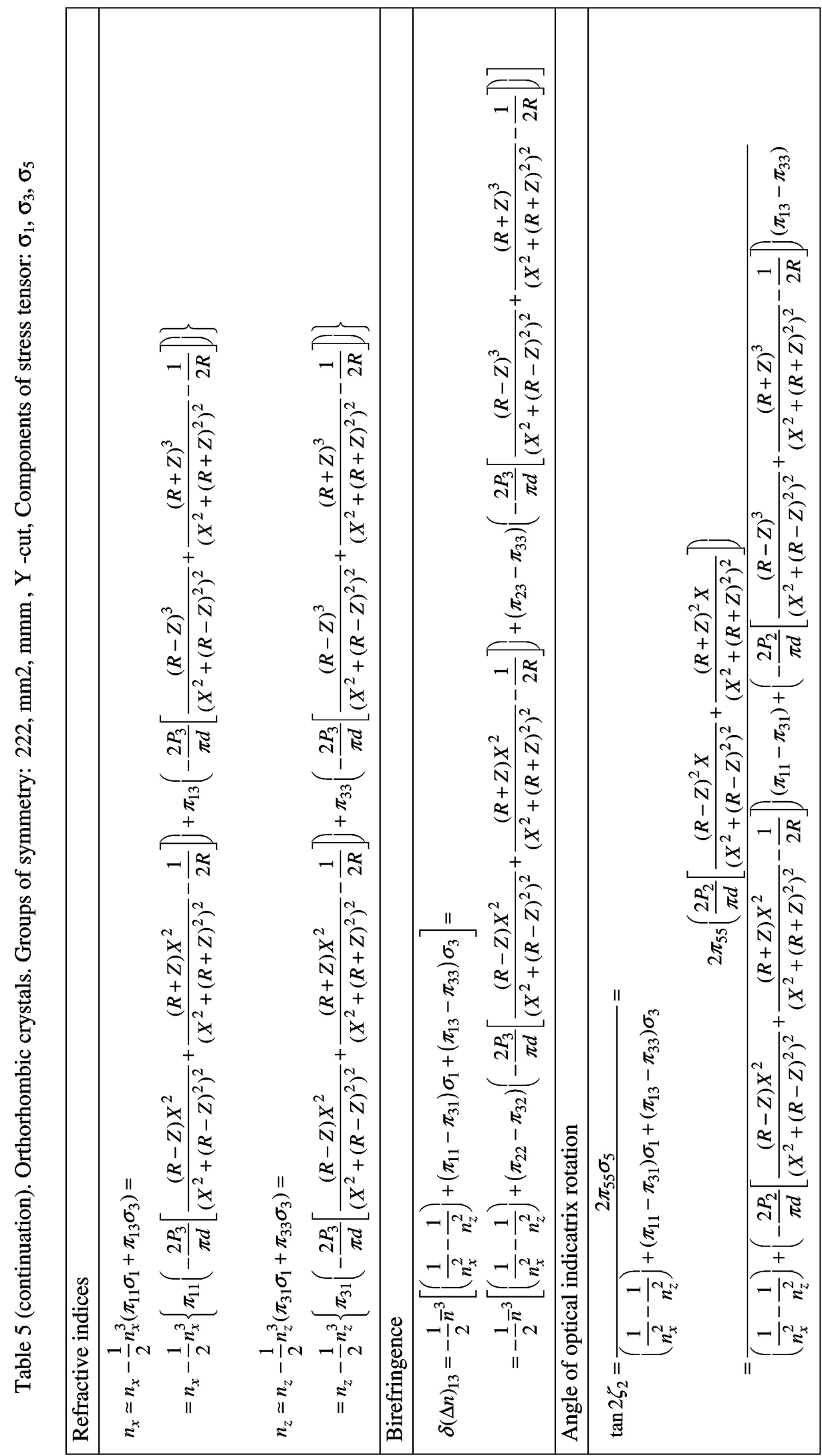




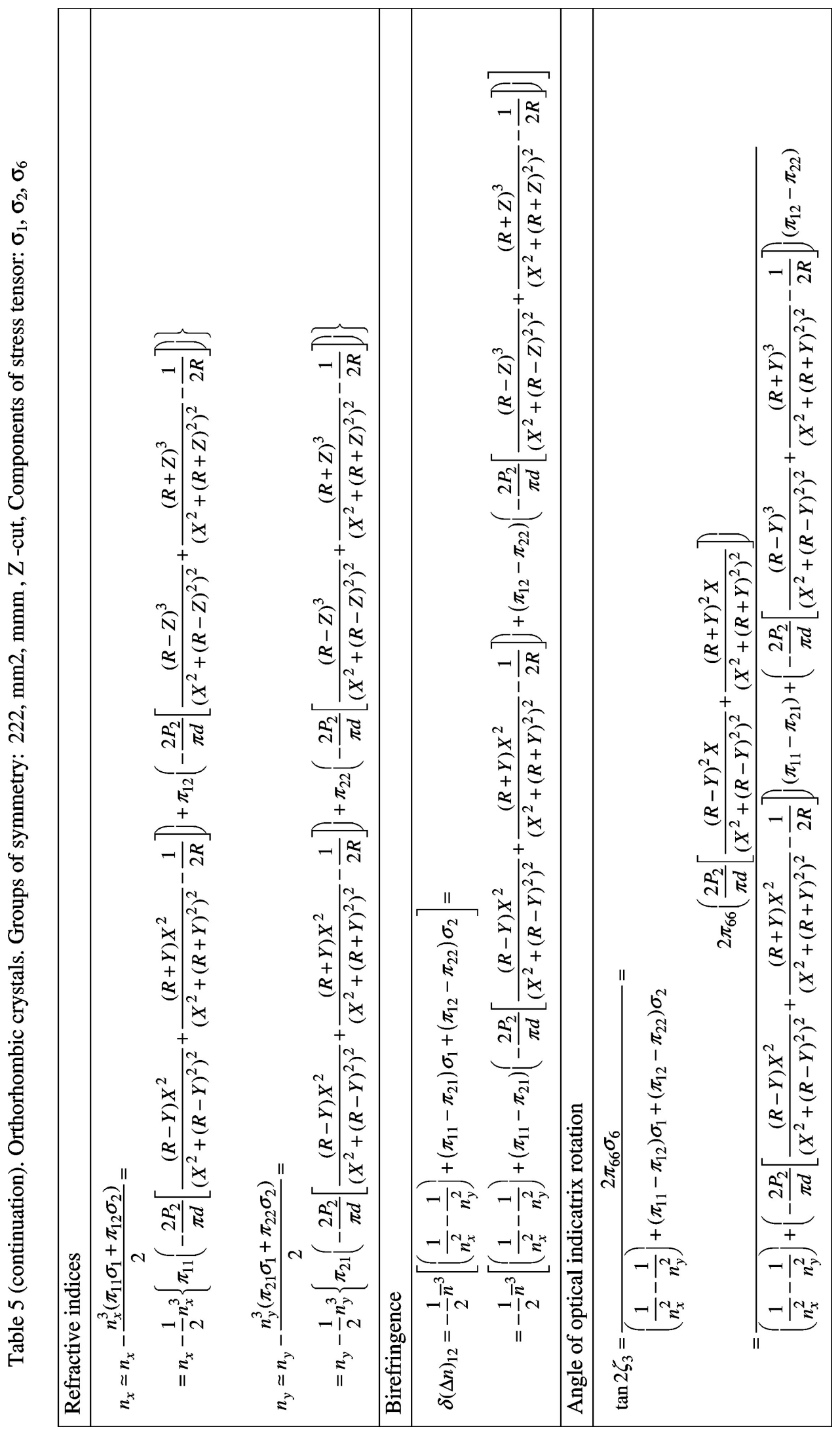

Ukr. J. Phys. Opt. 2012, V13, №2 
The denominator of Eq. (43) is equal to the term appearing in Eq. (42) in the square brackets. Hence, solving the system of these equations would result in determination of the coefficient $\pi_{44}$. The coefficients $\pi_{55}$ and $\pi_{66}$ may be evaluated using respectively the $Y$ - and $Z$-cut disks and applying the same procedures.

\subsection{Monoclinic and triclinic crystals}

Let us finally consider theoretical relations for the birefringence and the optical indicatrix rotation angle that hold for the low-symmetry monoclinic crystals (see Table 6). The birefringence increment for the $X$-cut disk under the force $P_{3}$ applied and the conditions $Z=0$ and $n_{2} \simeq n_{3}=\bar{n}$ takes the form

$$
\delta(\Delta n)_{23}=\bar{n}^{3} \frac{P_{3}}{\pi d} \frac{1}{2 R\left(Y^{2}+(R)^{2}\right)^{2}}\left[\left(\pi_{22}-\pi_{32}\right)\left(4 R^{2} Y^{2}-\left(Y^{2}+R^{2}\right)^{2}\right)+\left(\pi_{23}-\pi_{33}\right)\left(4 R^{4}-\left(Y^{2}+R^{2}\right)^{2}\right)\right],
$$

while the optical indicatrix rotation angle is given by the relation

$$
\tan 2 \zeta_{1}=\frac{-8 \pi_{44} R^{3} Y}{\left(4 R^{2} Y^{2}-\left(Y^{2}+R^{2}\right)^{2}\right)\left(\pi_{22}-\pi_{32}\right)+\left(4 R^{4}-\left(Y^{2}+R^{2}\right)^{2}\right)\left(\pi_{23}-\pi_{33}\right)} .
$$

Solving the system of Eqs. (44) and (45), one can find the piezooptic coefficient $\pi_{44}$. The coefficient $\pi_{55}$ is derived using the analogous procedure and the $Y$-cut disk.

Using the theoretical dependence of the birefringence on the $Y$ coordinate $(X=0)$ for the case of the force $P_{2}$ applied to the $Z$-cut disk,

$$
\delta(\Delta n)_{12}==\bar{n}^{3} \frac{P_{2}}{\pi d} \frac{1}{2 R\left(R^{2}-Y^{2}\right)}\left[\left(\pi_{12}-\pi_{22}\right)\left(4 R^{2}-R^{2}+Y^{2}\right)-\left(\pi_{11}-\pi_{21}\right)\left(R^{2}-Y^{2}\right)\right],
$$

and the corresponding relation for the angle of optical indicatrix rotation,

$$
\tan 2 \zeta_{3}=\frac{2\left\{\pi_{62}\left(4 R^{2}-R^{2}+Y^{2}\right)-\pi_{61}\left(R^{2}-Y^{2}\right)\right\}}{\left(4 R^{2}-R^{2}+Y^{2}\right)\left(\pi_{12}-\pi_{22}\right)-\left(R^{2}-Y^{2}\right)\left(\pi_{11}-\pi_{21}\right)},
$$

we can determine the combination $3 \pi_{62}-\pi_{61}$. For example, we have at $Y=0$

$$
\tan 2 \zeta_{3}=\frac{2\left\{3 \pi_{62}-\pi_{61}\right\}}{3\left(\pi_{12}-\pi_{22}\right)-\left(\pi_{11}-\pi_{21}\right)}, \delta(\Delta n)_{12}=\bar{n}^{3} \frac{P_{2}}{\pi d} \frac{1}{2 R}\left[3\left(\pi_{12}-\pi_{22}\right)-\left(\pi_{11}-\pi_{21}\right)\right] .
$$

Under the condition $Y=0$, the birefringence increment and the optical indicatrix rotation angle may be written respectively as

$$
\begin{aligned}
& \delta(\Delta n)_{12}=\bar{n}^{3} \frac{P_{2}}{\pi d} \frac{1}{2 R\left(X^{2}+(R)^{2}\right)^{2}} \times \\
& \times\left[\left(\pi_{11}-\pi_{21}\right)\left(4 R^{2} X^{2}-\left(X^{2}+R^{2}\right)^{2}\right)+\left(\pi_{12}-\pi_{22}\right)\left(4 R^{4}-\left(X^{2}+R^{2}\right)^{2}\right)-4 R^{3} X\left(\pi_{16}-\pi_{26}\right)\right] \\
& \tan 2 \zeta_{3}=\frac{2\left\{\pi_{61}\left(4 R^{2} X^{2}-\left(X^{2}+R^{2}\right)^{2}\right)+\pi_{62}\left(4 R^{4}-\left(X^{2}+R^{2}\right)^{2}\right)-4 R^{3} X \pi_{66}\right\}}{\left(4 R^{2} X^{2}-\left(X^{2}+R^{2}\right)^{2}\right)\left(\pi_{11}-\pi_{21}\right)+\left(4 R^{4}-\left(X^{2}+R^{2}\right)^{2}\right)\left(\pi_{12}-\pi_{22}\right)-4 R^{3} X\left(\pi_{16}-\pi_{26}\right)} .
\end{aligned}
$$

These relations enable evaluating only the piezooptic combination appearing in the nominator of Eq. (50), i.e. the term $\left\{\pi_{61}\left(4 R^{2} X^{2}-\left(X^{2}+R^{2}\right)^{2}\right)+\pi_{62}\left(4 R^{4}-\left(X^{2}+R^{2}\right)^{2}\right)-4 R^{3} X \pi_{66}\right\}$. In other words, it is impossible to find the $\pi_{66}$ coefficient itself for the case of monoclinic crystals. The similar is also true for the triclinic crystals (see Table 7): here the coefficients $\pi_{44}, \pi_{55}$ and $\pi_{66}$ cannot be determined separately. 


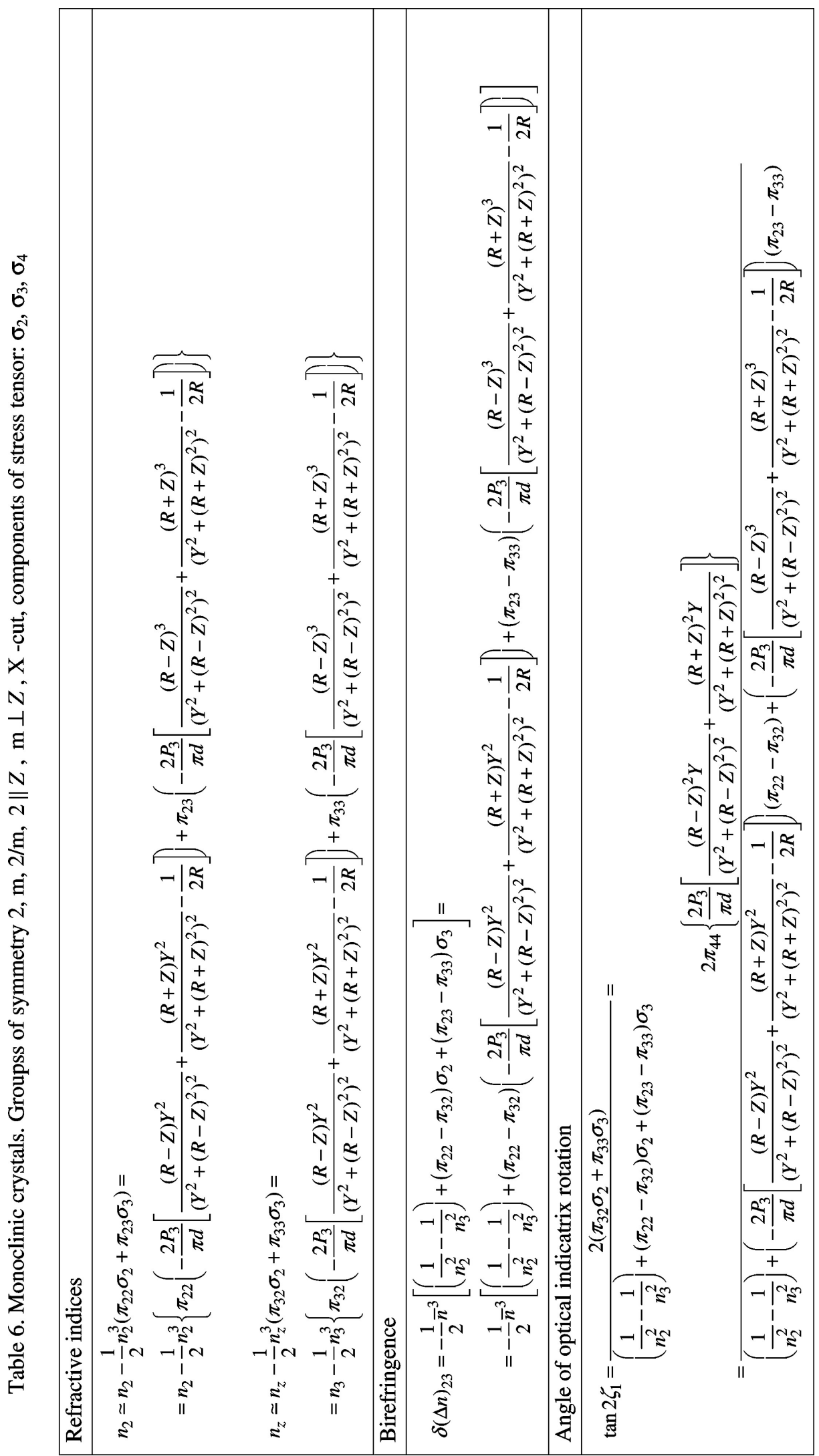

Ukr. J. Phys. Opt. 2012, V13, №2 


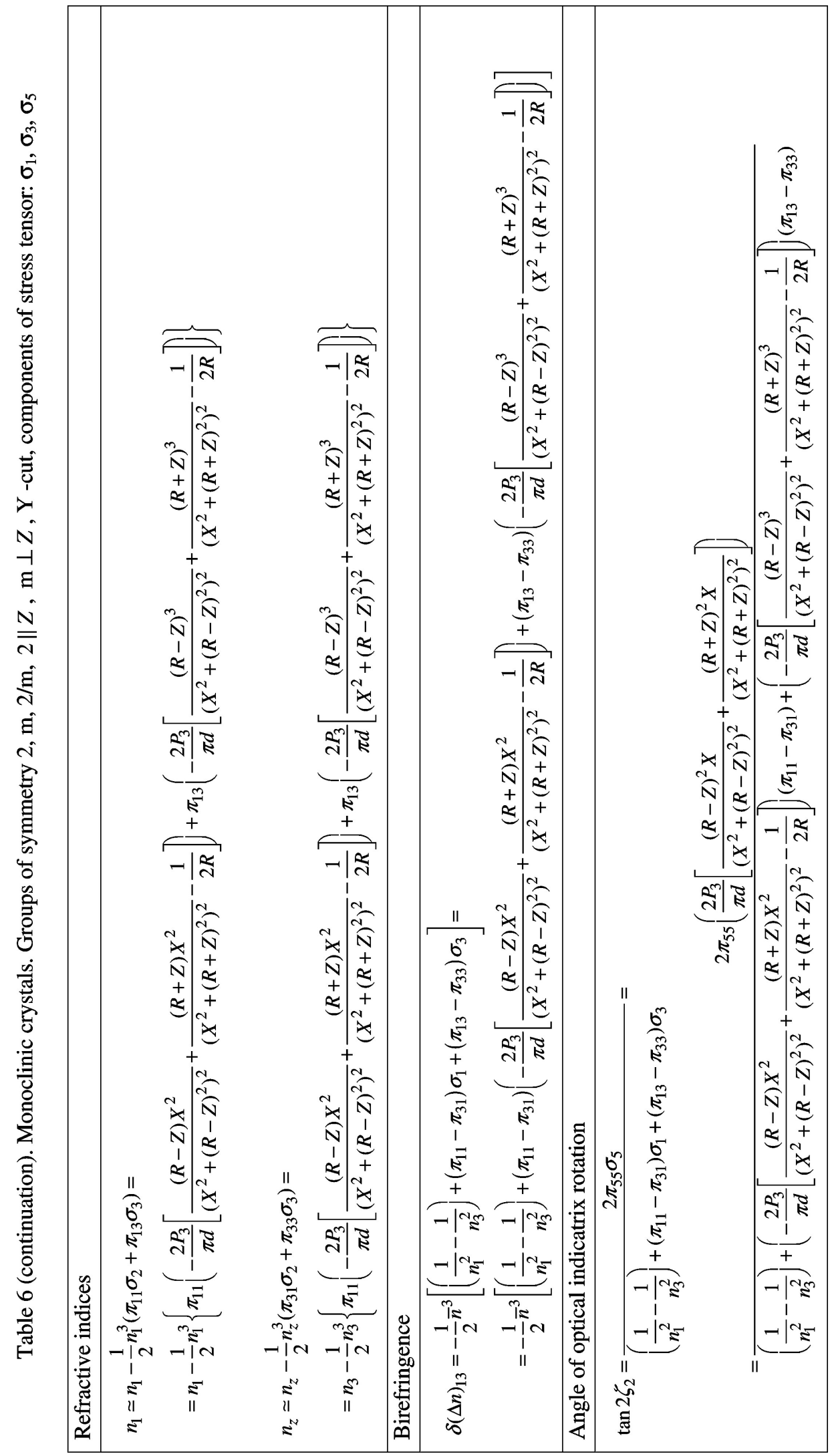




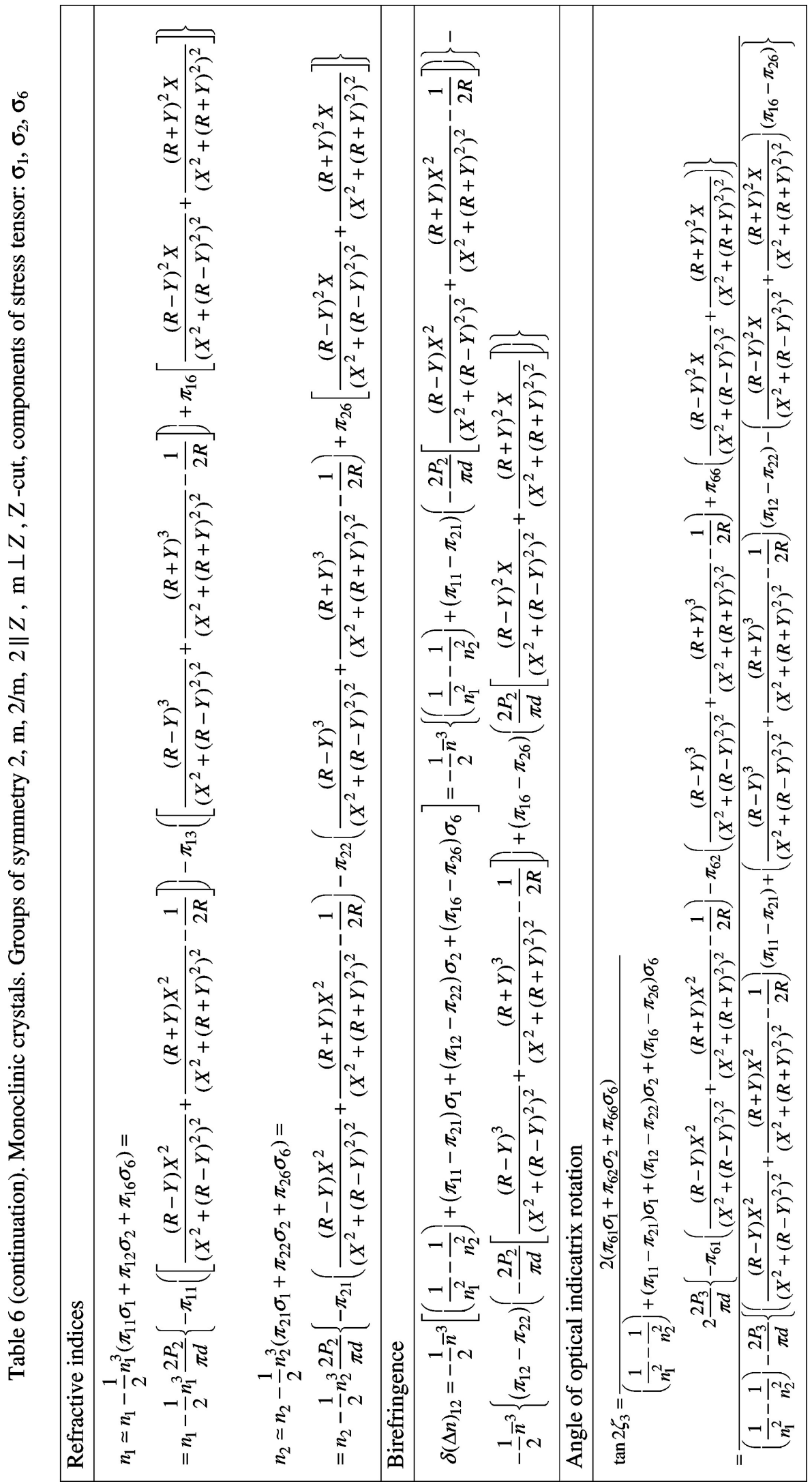

Ukr. J. Phys. Opt. 2012, V13, №2 


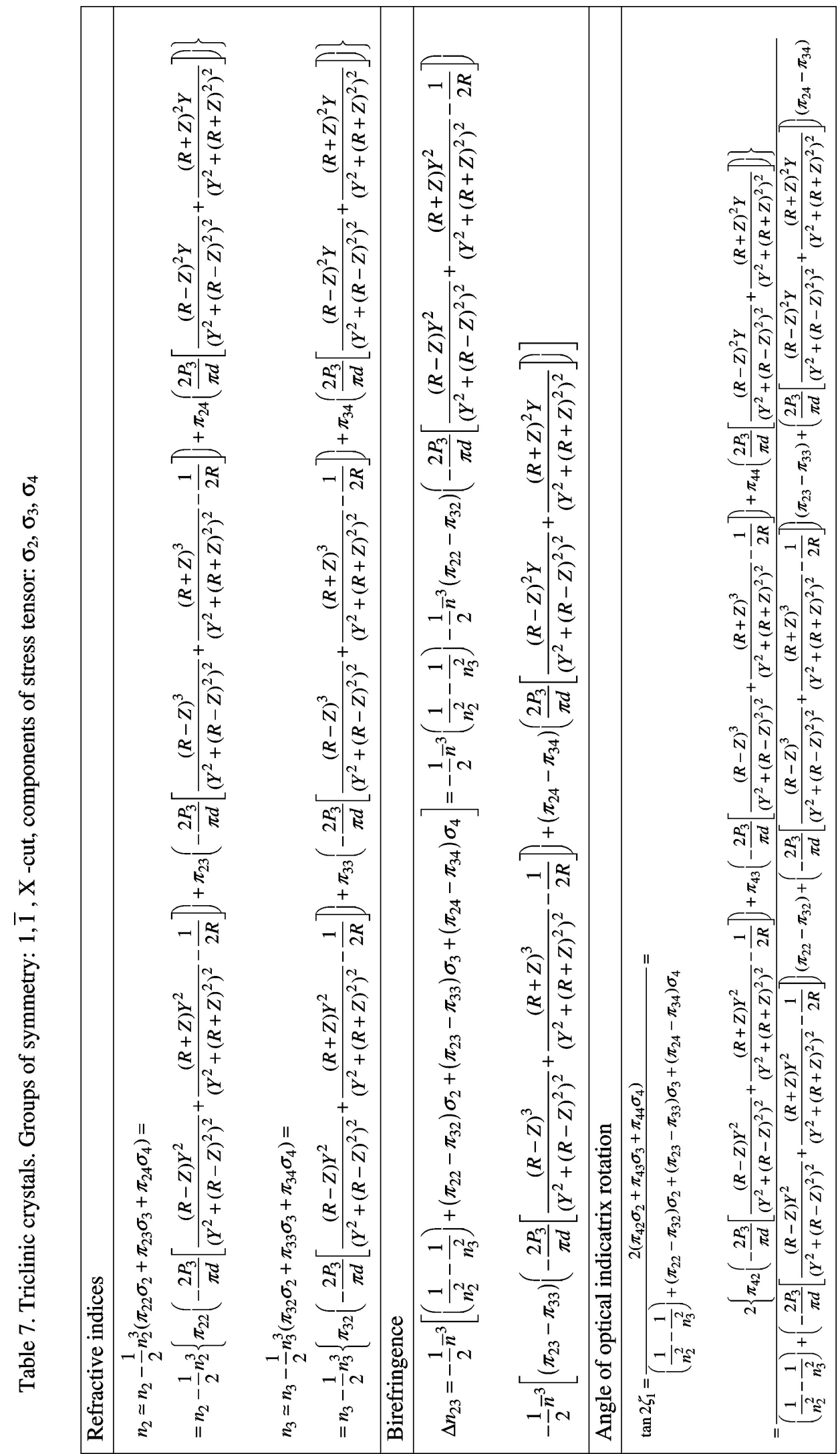




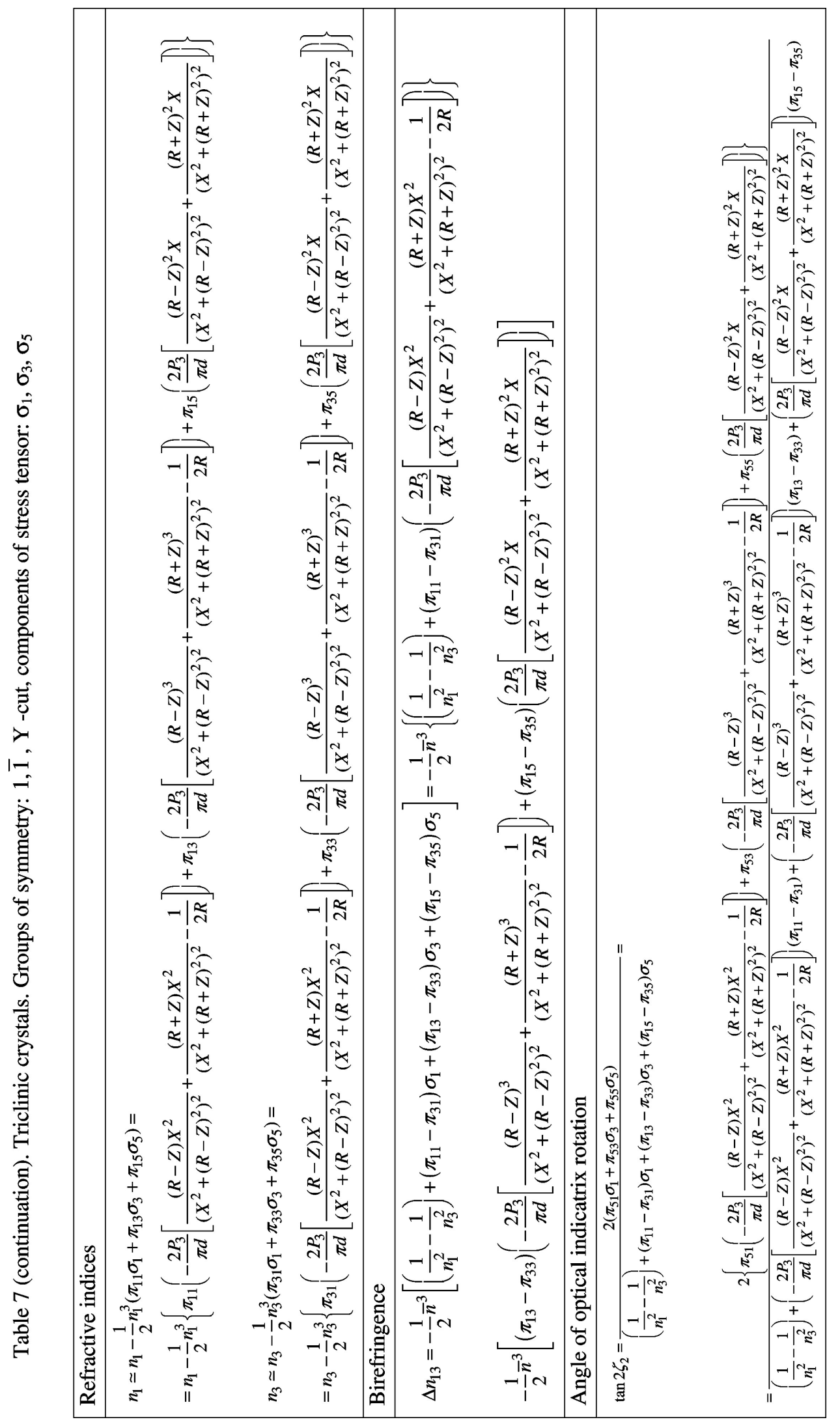

Ukr. J. Phys. Opt. 2012, V13, №2 


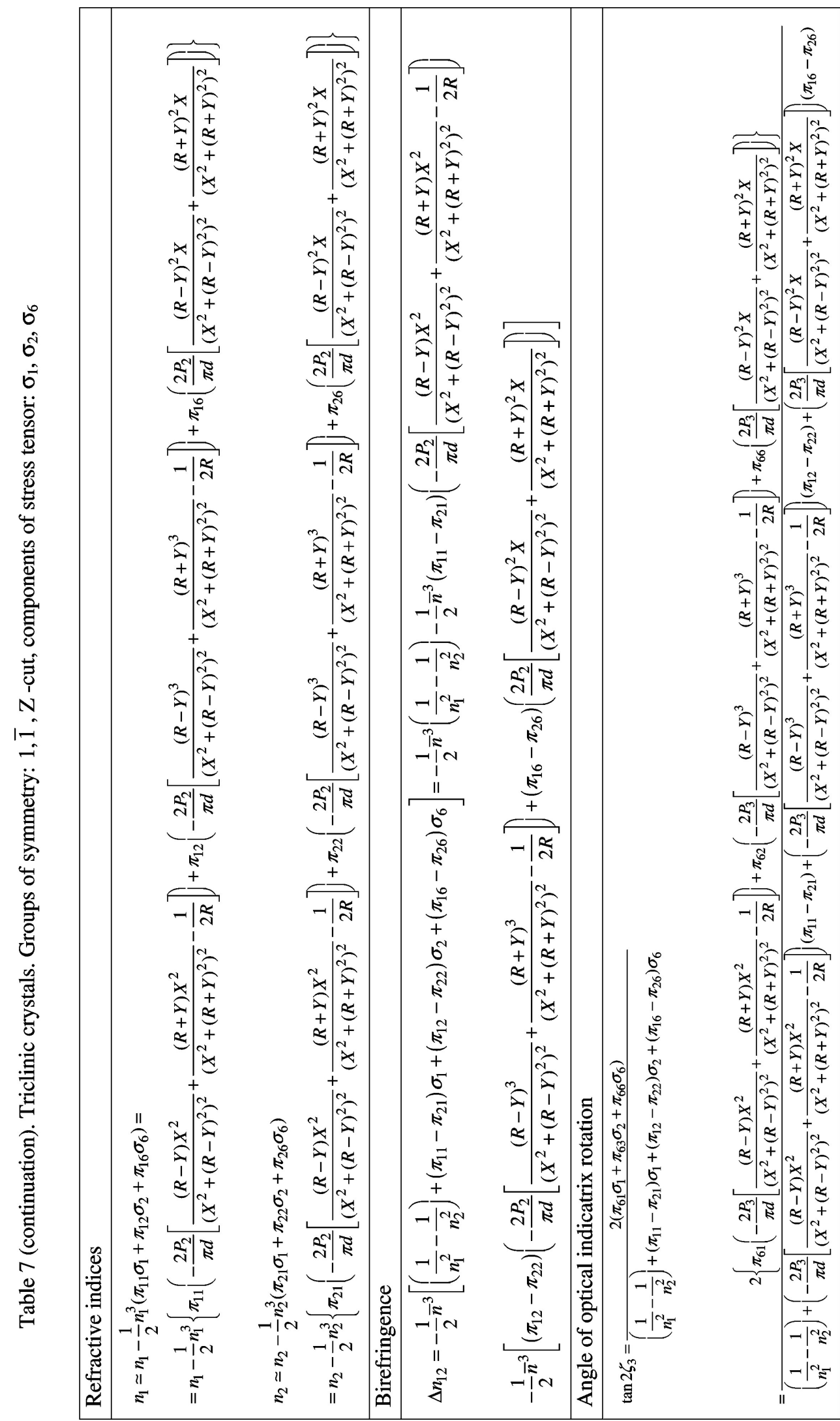




\section{Conclusion}

We have derived phenomenological relations that describe the changes in the optical birefringence and the optical indicatrix rotation angle for the crystals of all the point symmetry groups. These relations cover different geometries of mechanical stress application and different light propagation directions. In all of the cases analysed by us, we have dealt with crystalline disks compressed along their diameters. We have shown that the technique intended for the studies of piezooptic coefficients and developed in this work makes it possible to determine accurately the coefficients $\pi_{44}, \pi_{55}$ and $\pi_{66}$ for almost all of the point groups of symmetry.

\section{References}

1. Brewster D, 1815. Experiments on the depolarization of light as exhibited by various mineral, animal and vegetable bodies with a reference of the phenomena to the general principle of polarization. Phil. Trans. Roy. Soc. Lond. 105: 29-53.

2. Brewster $\mathrm{D}, 1816$. On the communication of the structure of doubly-refracting crystals to glass, murite of soda, flour spar, and other substances by mechanical compression and dilation. Phil. Trans. Roy. Soc. Lond. 106: 156-178.

3. Vasylkiv Yu, Kvasnyuk O, Krupych O, Mys O, Maksymuk O and Vlokh R, 2009. Reconstruction of 3D stress fields basing on piezooptic experiment. Ukr. J. Phys. Opt. 10: 2237.

4. Skab I, Smaga I, Savaryn V, Vasylkiv Y and Vlokh R, 2011. Torsion method for measuring piezooptic coefficients. Cryst. Res. Techn. 46: 23-36.

5. Krupych O, Savaryn V, Skab I and Vlokh R, 2011. Interferometric measurements of piezooptic coefficients by means of four-point bending method. Ukr. J. Phys. Opt. 12: 150-159.

6. Andrushchak A S, Bobitski Ya V, Kaidan M V, Mytsyk B G, Kityk A V and Schranz W, 2005. Two-fold interferometric measurements of piezo-optic constants: application to $\beta-\mathrm{BaB}_{2} \mathrm{O}_{4}$ crystals. Opt. Laser Technol. 37: 319-328.

7. Mys O, Adamiv V, Martynyuk-Lototska I, Burak Ya and Vlokh R, 2007. Piezooptic and acoustic properties of $\mathrm{KLiB}_{4} \mathrm{O}_{7}$ crystals. Ukr. J. Phys. Opt. 8: 138-142.

8. Frocht, Max Mark. Photoelasticity. New York: John Wiley (1946).

9. Ajmera P K, Huner B, Dutta A K and Hartley C S, 1988. Simulation and observation of infrared piezobirefringent images in diametrically compressed semiconductor disks. Appl. Opt. 27: $752-757$.

10. Vasylkiv Yu, Savaryn V, Smaga I, Krupych O, Skab I and Vlokh R, 2011. Studies of piezooptic coefficients in $\mathrm{LiNbO}_{3}$ crystals using a crystalline disk compressed along its diameter. Ukr. J. Phys. Opt. 12: 180-190.

Savaryn V., Skab I., Krupych O. and Vlokh R., 2012. The method for measuring piezooptic coefficients of crystals using a crystalline disk loaded along its diameter. Ukr.J.Phys.Opt. 13: 82 123

Анотація. В роботі розвинуто метод вимірювання n'єзооптичних коефіцієнтів $\pi_{k k}$ $(k=4,5,6)$ для кристалів, щуо належать майже до всіх точкових груп симетрії. Метод засновано на дослідженні подвійного оптичного заломлення $і$ кута повороту оптичної індикатриси за умови відомого просторового розподілу компонент тензора механічних напружень у кристалічному дискові, стиснутому вздовж діаметра. Представлено відповідні теоретичні співвідношення для опису п'єзооптичного ефекту в кристалах усіх груп симетрії.

Ukr. J. Phys. Opt. 2012, V13, №2 